\title{
Resolution Dimension Relative to Resolving Subcategories in Extriangulated Categories
}

\author{
Lingling Tan * $\mathbb{D}$ and Li Liu \\ School of Mathematical Sciences, Qufu Normal University, Qufu 273165, China; liuli618271@163.com \\ * Correspondence: tanll@qfnu.edu.cn
}

check for

updates

Citation: Tan, L.; Liu, L. Resolution Dimension Relative to Resolving Subcategories in Extriangulated Categories. Mathematics 2021, 9 , 980. https://doi.org/10.3390/math9090980

Academic Editor: Askar Tuganbaev

Received: 8 March 2021

Accepted: 19 April 2021

Published: 27 April 2021

Publisher's Note: MDPI stays neutral with regard to jurisdictional claims in published maps and institutional affiliations.

Copyright: (c) 2021 by the authors. Licensee MDPI, Basel, Switzerland. This article is an open access article distributed under the terms and conditions of the Creative Commons Attribution (CC BY) license (https:// creativecommons.org/licenses/by/ $4.0 /)$.

\begin{abstract}
Let $(\mathscr{C}, \mathbb{E}, \mathfrak{s})$ be an extriangulated category with a proper class $\xi$ of $\mathbb{E}$-triangles and $\mathfrak{X}$ a resolving subcategory of $\mathscr{C}$. In this paper, we introduce the notion of $\mathfrak{X}$-resolution dimension relative to the subcategory $\mathfrak{X}$ in $\mathscr{C}$, and then give some descriptions of objects with finite $\mathfrak{X}$-resolution dimension. In particular, we obtain Auslander-Buchweitz approximations for these objects. As applications, we construct adjoint pairs for two kinds of inclusion functors, and construct a new resolving subcategory from a given resolving subcategory which reformulates some known results.
\end{abstract}

Keywords: extriangulated categories; a proper class of $\mathbb{E}$-triangles; resolution dimension; resolving subcategories; Auslander-Buchweitz approximations

\section{Introduction}

In classical homological algebra, homological dimensions are important invariants, and every homological dimension is defined in terms of some certain subcategory. For example, one can define projective dimension in terms of the subcategory consisting of projective objects, and define injective dimension in terms of the subcategory of consisiting injective objects in any abelian category. Resolving subcategories play important roles in approximation theory (e.g., [1,2]). As an important example of resolving subcategories, Auslander and Buchweitz [3] studied the approximation theory of the subcategory consisting of maximal Cohen-Macaulay modules over an Artin algebra. Zhu [4] studied the resolution dimension with respect to a resolving subcategory in an abelian category, and Huang [5] introduced relative preresolving subcategories in an abelian category and defined homological dimensions relative to these subcategories. In [6,7], Ma, Zhao, and Huang investigated homological dimensions relative to (pre)resolving subcategories in triangulated categories with a proper class of triangles. For more references on resolution and homological dimension, see [8-11], for example.

Exact and triangulated categories are two important structures in category theory. In [12], Nakaoka and Palu introduced the notion of extriangulated categories as a simultaneous generalization of exact categories and extension-closed subcategories of triangulated categories. After that, the study of extriangulated categories has become an active topic, and up to now, many results on exact categories and triangulated categories can be unified in the same framework, e.g., see [8,12-16]. Recently, Hu, Zhang, Zhou [13] studied a relative homological algebra in an extriangulated category $(\mathscr{C}, \mathbb{E}, \mathfrak{s})$ which parallels the relative homological algebra in triangulated categories and exact categories. By specifying a class of $\mathbb{E}$-triangles, which is called a proper class $\xi$ of $\mathbb{E}$-triangles, the authors introduced $\xi$-projective, $\xi$-injective, $\xi$-G projective and $\xi$-Ginjective dimensions, and discussed their properties. In abelian categories, the subcategory consisting of Gorenstein projective objects is a resolving subcategory, thus the aim of this paper is to introduce a notion of resolving subcategories in extriangulated categories, which regards the subcategory consisting of $\xi-\mathcal{G}$ projective objects as a special example. After this, we devote to further studying homological dimensions relative to a resolving subcategory in extriangulated categories which 
recovers lots of known results in abelian and triangulated categories, and is new in exact categories. The paper is organized as follows.

In Section 2, we give some terminology and some preliminary results. In particular, we introduce the notion of resolving subcategories in extriangulated categories with a proper class of $\mathbb{E}$-triangles.

In Section 3, we introduce the notion of $\mathfrak{X}$-resolution dimension of objects relative to a resolving subcategory $\mathfrak{X}$, and some homological properties of resolution dimension are obtained. In particular, we obtain Auslander-Buchweitz approximation $\mathbb{E}$-triangles (see Proposition 4) for objects with finite $\mathfrak{X}$-resolution dimension. Our main result is the following.

Theorem 1. Let $\mathfrak{X}$ be a resolving subcategory of an extriangulated category $(\mathscr{C}, \mathbb{E}, \mathfrak{s})$ with a proper class of $\mathbb{E}$-triangles $\xi$, and $\mathfrak{H}$ a $\boldsymbol{\xi}$-cogenerator of $\mathfrak{X}$ with $\mathfrak{X} \perp \mathfrak{H}$. Let $\mathfrak{X}$ be the full subcategory of $\mathscr{C}$ whose objects have finite $\mathfrak{X}$-resolution dimension, and let $\Omega_{\mathfrak{X}}^{n}(M)$ (resp. $\Omega^{n}(M)$ ) be an nth $\mathfrak{X}$-syzygy (resp. syzygy) of $M$. Assume that one of the following conditions satisfies:

(a) $\mathfrak{H}$ is closed under cocones of $\xi$-deflations.

(b) $\mathfrak{H}$ is closed under direct summands.

For any $M \in \mathscr{C}$, if $M \in \widehat{\mathfrak{X}}$, then the following statements are equivalent:

(1) $\mathfrak{X}$-res.dim $M \leq m$.

(2) $\Omega^{n}(M) \in \mathfrak{X}$ for all $n \geq m$.

(3) $\Omega_{\mathfrak{X}}^{n}(M) \in \mathfrak{X}$ for all $n \geq m$.

(4) $\xi x t_{\tilde{\xi}}^{n}(M, H)=0$ for all $n>m$ and all $H \in \mathfrak{H}$.

(5) $\xi x t_{\tilde{\zeta}}^{n}(M, L)=0$ for all $n>m$ and all $L \in \widehat{\mathfrak{H}}$.

(6) $\quad M$ admits a right $\mathfrak{X}$-approximation $\varphi: X \rightarrow M$, where $\varphi$ is a $\xi$-defaltion, such that there is an $\mathbb{E}$-triangle $K \longrightarrow X \stackrel{\varphi}{\longrightarrow} M-\rightarrow$ satisfying $\mathfrak{H}$-res.dim $K \leq m-1$.

(7) There are two $\mathbb{E}$-triangles

$$
W_{M} \rightarrow X_{M} \rightarrow M->
$$

and

$$
M \rightarrow W^{M} \longrightarrow X^{M}->
$$

in $\xi$ such that $X_{M}$ and $X^{M}$ are in $\mathfrak{X}$ and $\mathfrak{H}$-res.dim $W_{M} \leq m-1, \mathfrak{H}$-res.dim $W^{M}=$ $\mathfrak{X}$-res.dim $W^{M} \leq m$.

As applications, in Section 4, we will further study objects with a finite resolution dimension with respect to a resolving subcategory $\mathfrak{X}$. We construct adjoint pairs for two kinds of inclusion functors (see Theorems 3 and 4 ). Given a resolving subcategory $\mathfrak{X}$ of $\mathscr{C}$, we construct a new resolving subcategory $\mathcal{G} \mathcal{P}_{\mathfrak{X}}(\xi)$ with a $\xi$-cogenerator $\mathfrak{X} \cap \perp \mathfrak{X}$ (see Theorem 5), which generalizes the Gorenstein projective subcategory $\mathcal{G} \mathcal{P}(\xi)$ given by $\mathrm{Hu}$, Zhang, and Zhou [13] of [Definition 4.8].

Throughout this paper, all subcategories are full, additive and closed under isomorphisms.

\section{Preliminaries}

We first recall some notions and some needed properties of extriangulated categories from [12].

Let $\mathscr{C}$ be an additive category and $\mathbb{E}: \mathscr{C}$ op $\times \mathscr{C} \rightarrow \mathfrak{A} b$ a biadditive functor, where $\mathfrak{A} b$ is the category of abelian groups. Let $A, C \in \mathscr{C}$. An element $\delta \in \mathbb{E}(C, A)$ is called an $\mathbb{E}$-extension. Two sequences of morphisms

$$
A \stackrel{x}{\rightarrow} B \stackrel{y}{\rightarrow} C \text { and } A \stackrel{x^{\prime}}{\rightarrow} B^{\prime} \stackrel{y^{\prime}}{\rightarrow} C
$$


are said to be equivalent if there exists an isomorphism $b \in \operatorname{Hom}_{\mathscr{C}}\left(B, B^{\prime}\right)$ such that $x^{\prime}=b x$ and $y=y^{\prime} b$. We denote by $[A \stackrel{x}{\rightarrow} B \stackrel{y}{\rightarrow} C]$ the equivalence class of $A \stackrel{x}{\longrightarrow} B \stackrel{y}{\longrightarrow} C$. In particular, we write $0:=\left[A \stackrel{\left(\begin{array}{c}\mathrm{Id}_{A} \\ 0\end{array}\right.}{\longrightarrow} A \oplus C \stackrel{\left(0 \mathrm{Id}_{C}\right)}{\longrightarrow} C\right]$.

For an $\mathbb{E}$-extension $\delta \in \mathbb{E}(C, A)$, we briefly write

$$
a_{*} \delta:=\mathbb{E}(C, a)(\delta) \text { and } c^{*} \delta:=\mathbb{E}(c, A)(\delta) .
$$

For two $\mathbb{E}$-extensions $\delta \in \mathbb{E}(C, A)$ and $\delta^{\prime} \in \mathbb{E}\left(C^{\prime}, A^{\prime}\right)$, a morphism from $\delta$ to $\delta^{\prime}$ is a pair $(a, c)$ of morphisms with $a \in \operatorname{Hom}_{\mathscr{C}}\left(A, A^{\prime}\right)$ and $c \in \operatorname{Hom}_{\mathscr{C}}\left(C, C^{\prime}\right)$ such that $a_{*} \delta=c^{*} \delta^{\prime}$.

Definition 1. ([12] of [Definition 2.9], [17]) Let $\mathfrak{s}$ be a correspondence which associates an equivalence class $\mathfrak{s}(\delta)=[A \stackrel{x}{\rightarrow} B \stackrel{y}{\rightarrow} C]$ to each $\mathbb{E}$-extension $\delta \in \mathbb{E}(C, A)$. The correspondence $\mathfrak{s}$ is called $a$ realization of $\mathbb{E}$ provided that it satisfies the following condition.

(R) Let $\delta \in \mathbb{E}(C, A)$ and $\delta^{\prime} \in \mathbb{E}\left(C^{\prime}, A^{\prime}\right)$ be any pair of $\mathbb{E}$-extensions with

$$
\mathfrak{s}(\delta)=[A \stackrel{x}{\rightarrow} B \stackrel{y}{\rightarrow} C] \text { and } \mathfrak{s}\left(\delta^{\prime}\right)=\left[A^{\prime} \stackrel{x^{\prime}}{\rightarrow} B^{\prime} \stackrel{y^{\prime}}{\rightarrow} C^{\prime}\right] .
$$

Then for any morphism $(a, c): \delta \rightarrow \delta^{\prime}$, there exists $b \in \operatorname{Hom}_{\mathscr{C}}\left(B, B^{\prime}\right)$ such that the following diagram

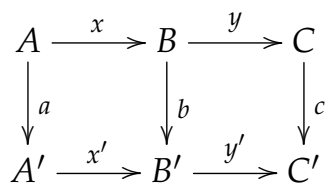

commutes.

Let $\mathfrak{s}$ be a realization of $\mathbb{E}$. If $\mathfrak{s}(\delta)=[A \stackrel{x}{\rightarrow} B \stackrel{y}{\rightarrow} C]$ for some $\mathbb{E}$-extension $\delta \in \mathbb{E}(C, A)$, then we say that the sequence $A \stackrel{x}{\rightarrow} B \stackrel{y}{\rightarrow} C$ realizes $\delta$; and in the condition $(R)$, we say that the triple $(a, b, c)$ realizes the morphism $(a, c)$.

For any two equivalence classes $[A \stackrel{x}{\rightarrow} B \stackrel{y}{\rightarrow} C]$ and $\left[A^{\prime} \stackrel{x^{\prime}}{\rightarrow} B^{\prime} \stackrel{y^{\prime}}{\rightarrow} C^{\prime}\right]$, we define

$$
[A \stackrel{x}{\rightarrow} B \stackrel{y}{\longrightarrow} C] \oplus\left[A^{\prime} \stackrel{x^{\prime}}{\longrightarrow} B^{\prime} \stackrel{y^{\prime}}{\longrightarrow} C^{\prime}\right]:=\left[A \oplus A^{\prime} \stackrel{x \oplus x^{\prime}}{\longrightarrow} B \oplus B^{\prime} \stackrel{y \oplus y^{\prime}}{\longrightarrow} C \oplus C^{\prime}\right] .
$$

Definition 2. ([12] of [Definition 2.10], [17]) A realization $\mathfrak{s}$ of $\mathbb{E}$ is called additive if it satisfies the following conditions.

(1) For any $A, C \in \mathscr{C}$, the split $\mathbb{E}$-extension $0 \in \mathbb{E}(C, A)$ satisfies $\mathfrak{s}(0)=0$.

(2) For any pair of $\mathbb{E}$-extensions $\delta \in \mathbb{E}(C, A)$ and $\delta^{\prime} \in \mathbb{E}\left(C^{\prime}, A^{\prime}\right)$, we have $\mathfrak{s}\left(\delta \oplus \delta^{\prime}\right)=$ $\mathfrak{s}(\delta) \oplus \mathfrak{s}\left(\delta^{\prime}\right)$.

Definition 3. ([12] of [Definition 2.12], [17]) The triple $(\mathscr{C}, \mathbb{E}, \mathfrak{s})$ is called an externally triangulated (or extriangulated for short) category if it satisfies the following conditions.

$(E T 1) \mathbb{E}: \mathscr{C}^{\mathrm{op}} \times \mathscr{C} \rightarrow \mathfrak{A} b$ is a biadditive functor.

$(E T 2) \mathfrak{s}$ is an additive realization of $\mathbb{E}$.

(ET3)Let $\delta \in \mathbb{E}(C, A)$ and $\delta^{\prime} \in \mathbb{E}\left(C^{\prime}, A^{\prime}\right)$ be any pair of $\mathbb{E}$-extensions with

$$
\mathfrak{s}(\delta)=[A \stackrel{x}{\longrightarrow} B \stackrel{y}{\longrightarrow} C] \text { and } \mathfrak{s}\left(\delta^{\prime}\right)=\left[A^{\prime} \stackrel{x^{\prime}}{\longrightarrow} B^{\prime} \stackrel{y^{\prime}}{\longrightarrow} C^{\prime}\right] .
$$

For any commutative diagram 


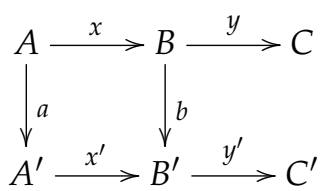

in $\mathscr{C}$, there exists a morphism $(a, c): \delta \rightarrow \delta^{\prime}$ which is realized by the triple $(a, b, c)$.

(ET3) ${ }^{\text {op }}$ Dual of (ET3).

(ET4)Let $\delta \in \mathbb{E}(C, A)$ and $\rho \in \mathbb{E}(F, B)$ be any pair of $\mathbb{E}$-extensions with

$$
\mathfrak{s}(\delta)=[A \stackrel{x}{\rightarrow} B \stackrel{y}{\rightarrow} C] \text { and } \mathfrak{s}(\rho)=[B \stackrel{u}{\longrightarrow} D \stackrel{v}{\rightarrow} F] .
$$

Then there exist an object $E \in \mathscr{C}$, an $\mathbb{E}$-extension $\xi$ with $\mathfrak{s}(\xi)=[A \stackrel{z}{\rightarrow} D \stackrel{w}{\longrightarrow} E]$, and $a$ commutative diagram

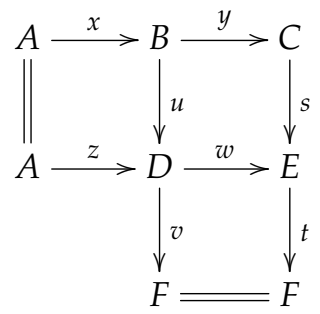

in $\mathscr{C}$, which satisfy the following compatibilities.

(i) $\mathfrak{s}\left(y_{*} \rho\right)=[C \stackrel{s}{\rightarrow} E \stackrel{t}{\rightarrow} F]$.

(ii) $s^{*} \xi=\delta$.

(iii) $x_{*} \xi=t^{*} \rho$.

(ET4) $)^{\text {op }}$ Dual of (ET4).

Remark 1. Please note that both exact categories and triangulated categories are extriangulated categories (see [12] of [Proposition 3.22]) and extension closed subcategories of extriangulated categories are again extriangulated (see [12] of [Remark 2.18]). Moreover, there exist extriangulated categories which are neither exact categories nor triangulated categories (see [12] of [Proposition 3.30] and [13] of [Remark 3.3]).

We will use the following terminology.

Definition 4. ([12] of [Definitions 2.15 and 2.19], [17]) Let $(\mathscr{C}, \mathbb{E}, \mathfrak{s})$ be an extriangulated category.

1. A sequence $A \stackrel{x}{\rightarrow} B \stackrel{y}{\rightarrow} C$ is called a conflation if it realizes some $\mathbb{E}$-extension $\delta \in \mathbb{E}(C, A)$. In this case, $x$ is called an inflation and $y$ is called a deflation.

2. If a conflation $A \stackrel{x}{\rightarrow} B \stackrel{y}{\rightarrow} C$ realizes $\delta \in \mathbb{E}(C, A)$, we call the pair $(A \stackrel{x}{\rightarrow} B \stackrel{y}{\rightarrow} C, \delta)$ an $\mathbb{E}$-triangle, and write it in the following way.

$$
A \stackrel{x}{\rightarrow} B \stackrel{y}{\longrightarrow} C^{-~} \stackrel{\delta}{\rightarrow}
$$

We usually do not write this " $\delta$ " if it is not used in the argument.

3. Let $A \stackrel{x}{\longrightarrow} B \stackrel{y}{\longrightarrow} C \stackrel{\delta}{\rightarrow}$ and $A^{\prime} \stackrel{x^{\prime}}{\longrightarrow} B^{\prime} \stackrel{y^{\prime}}{\longrightarrow} C^{\prime} \stackrel{\delta^{\prime}}{\rightarrow}$ be any pair of $\mathbb{E}$-triangles. If a triplet $(a, b, c)$ realizes $(a, c): \delta \rightarrow \delta^{\prime}$, then we write it as

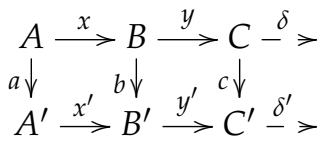

and call $(a, b, c)$ a morphism of $\mathbb{E}$-triangles.

If $a, b, c$ above are isomorphisms, then $A \stackrel{x}{\rightarrow} B \stackrel{y}{\rightarrow} C \stackrel{\delta}{\rightarrow}$ and $A^{\prime} \stackrel{x^{\prime}}{\rightarrow} B^{\prime} \stackrel{y^{\prime}}{\rightarrow} C^{\prime} \stackrel{\delta^{\prime}}{\rightarrow}$ are said to be isomorphic. 
Remark 2. We can view the collection of all $\mathbb{E}$-triangles together with morphisms of $\mathbb{E}$-triangles as an additive category. Indeed,

(i) Let $(a, b, c)$ be a morphism from $A \stackrel{x}{\rightarrow} B \stackrel{y}{\rightarrow} C \stackrel{\delta}{\rightarrow}$ to $A^{\prime} \stackrel{x^{\prime}}{\rightarrow} B^{\prime} \stackrel{y^{\prime}}{\rightarrow} C^{\prime} \stackrel{\delta^{\prime}}{\rightarrow}$, and let $\left(a^{\prime}, b^{\prime}, c^{\prime}\right)$ be a morphism from $A^{\prime} \stackrel{x^{\prime}}{\rightarrow} B^{\prime} \stackrel{y^{\prime}}{\rightarrow} C^{\prime} \stackrel{\delta^{\prime}}{\rightarrow}$ to $A^{\prime \prime} \stackrel{x^{\prime \prime}}{\rightarrow} B^{\prime \prime} \stackrel{y^{\prime \prime}}{\rightarrow} C^{\prime \prime} \stackrel{\delta^{\prime \prime}}{\rightarrow}$. The composition is defined by $\left(a^{\prime} a, b^{\prime} b, c^{\prime} c\right)$.

The composition is well defined. In fact, assume that $(a, c): \delta \rightarrow \delta^{\prime}$ and $\left(a^{\prime}, c^{\prime}\right): \delta^{\prime} \rightarrow \delta^{\prime \prime}$ define morphisms of $\mathbb{E}$-extensions, then $a_{*} \delta=c^{*} \delta^{\prime}$ and $a^{\prime}{ }_{*} \delta^{\prime}=c^{\prime *} \delta^{\prime \prime}$. Thus,

$$
\begin{aligned}
\left(a^{\prime} a\right)_{*} \delta & =\left(a^{\prime}{ }_{*} a_{*}\right) \delta=a^{\prime}{ }_{*}\left(a_{*} \delta\right)=a^{\prime}{ }_{*}\left(c^{*} \delta^{\prime}\right)=\left(a^{\prime}{ }_{*} c^{*}\right) \delta^{\prime} \\
& =\left(c^{*} a^{\prime}{ }_{*}\right) \delta^{\prime}=c^{*}\left(a^{\prime}{ }_{*} \delta^{\prime}\right)=c^{*}\left(c^{\prime *} \delta^{\prime \prime}\right)=\left(c^{*} c^{\prime *}\right) \delta^{\prime \prime}=\left(c^{\prime} c\right)^{*} \delta^{\prime \prime},
\end{aligned}
$$

that is, $\left(a^{\prime} a, c^{\prime} c\right): \delta \rightarrow \delta^{\prime \prime}$ is a morphism of $\mathbb{E}$-extensions.

(ii) For an $\mathbb{E}$-triangle $A \stackrel{x}{\longrightarrow} B \stackrel{y}{\rightarrow} C^{-\delta} \rightarrow$, the identity morphism is $\left(\operatorname{Id}_{A}, \operatorname{Id}_{B}, \operatorname{Id}_{C}\right)$.

(iii) The associativity of the composition is inherited by the associativity of the composition in $\mathscr{C}$.

(iv) The E-triangle $0 \stackrel{0}{\rightarrow} 0 \stackrel{0}{\rightarrow} 0{ }_{-}^{0} \rightarrow$ is an initial and terminal object.

(v) For objects $X, Y$ in a category, write $\iota_{X}: X \rightarrow X \sqcup Y$ and $\iota_{Y}: Y \rightarrow X \sqcup Y$ for the morphisms equipping the coproduct (if it exists), and $\pi_{X}: X \sqcap Y \rightarrow X$ and $\pi_{Y}: X \sqcap Y \rightarrow$ $Y$ for the morphisms equipping the product (if it exists). Now since $\mathscr{C}$ is additive, there is an isomorphism $\varphi_{X, Y}: X \sqcup Y \rightarrow X \sqcap Y$. Now fix objects $A \stackrel{x}{\rightarrow} B \stackrel{y}{\rightarrow} C \stackrel{\delta}{\rightarrow}$ and $A^{\prime} \stackrel{x^{\prime}}{\longrightarrow} B^{\prime} \stackrel{y^{\prime}}{\longrightarrow} C^{\prime} \stackrel{\delta^{\prime}}{\rightarrow}$. There are isomorphisms

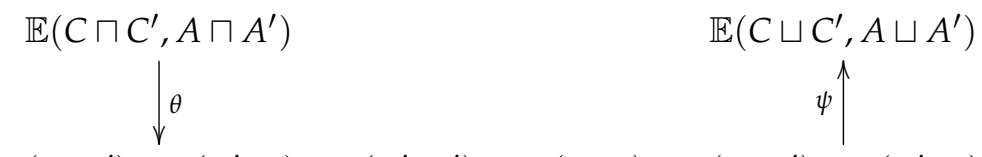

$\mathbb{E}(C, A) \sqcap \mathbb{E}\left(C, A^{\prime}\right) \sqcap \mathbb{E}\left(C^{\prime}, A\right) \sqcap \mathbb{E}\left(C^{\prime}, A^{\prime}\right)=\mathbb{E}(C, A) \sqcup \mathbb{E}\left(C, A^{\prime}\right) \sqcup \mathbb{E}\left(C^{\prime}, A\right) \sqcup \mathbb{E}\left(C^{\prime}, A^{\prime}\right)$

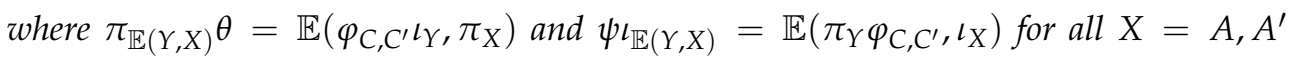
and $Y=C, C^{\prime}$. Now let $\delta \sqcup \delta^{\prime}=\psi(\kappa)$ and $\delta \sqcap \delta^{\prime}=\theta^{-1}(\kappa)$ where $\kappa=\iota_{\mathbb{E}(C, A)}(\delta)+$ $\iota_{\mathbb{E}\left(C^{\prime}, A^{\prime}\right)}\left(\delta^{\prime}\right)$. Then it is easy to check: that

$$
A \sqcup A^{\prime} \stackrel{x \sqcup x^{\prime}}{\longrightarrow} B \sqcup B^{\prime} \stackrel{y \sqcup y^{\prime}}{\longrightarrow} C \sqcup C^{\prime} \stackrel{\delta \sqcup \delta^{\prime}}{\rightarrow}
$$

is the coproduct of $A \stackrel{x}{\rightarrow} B \stackrel{y}{\rightarrow} C^{-} \stackrel{\delta}{\rightarrow}$ and $A^{\prime} \stackrel{x^{\prime}}{\rightarrow} B^{\prime} \stackrel{y^{\prime}}{\rightarrow} C^{\prime} \stackrel{\delta^{\prime}}{\rightarrow}$; and that

$$
A \sqcap A^{\prime} \stackrel{x \sqcap x^{\prime}}{\longrightarrow} B \sqcap B^{\prime} \stackrel{y \sqcap y^{\prime}}{\longrightarrow} C \sqcap C^{\prime} \stackrel{\delta \sqcap \delta^{\prime}}{\longrightarrow}
$$

is the product of $A \stackrel{x}{\rightarrow} B \stackrel{y}{\rightarrow} C \stackrel{-\delta}{\rightarrow}$ and $A^{\prime} \stackrel{x^{\prime}}{\rightarrow} B^{\prime} \stackrel{y^{\prime}}{\rightarrow} C^{\prime} \stackrel{\delta^{\prime}}{\rightarrow}$; and that the triple $\left(\varphi_{A, A^{\prime}}, \varphi_{B, B^{\prime}}, \varphi_{C, C^{\prime}}\right)$ is the (unique) morphism

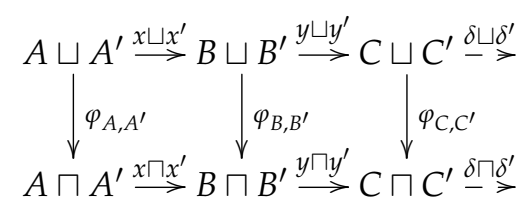

induced by the universal property.

The following condition is analogous to the weak idempotent completeness in exact categories (see [12] of [Condition 5.8]).

Condition (WIC) Consider the following conditions. 
(a) Let $f \in \mathscr{C}(A, B), g \in \mathscr{C}(B, C)$ be any composable pair of morphisms. If $g f$ is an inflation, then so is $f$.

(b) Let $f \in \mathscr{C}(A, B), g \in \mathscr{C}(B, C)$ be any composable pair of morphisms. If $g f$ is a deflation, then so is $g$.

Example 1. (1) If $\mathscr{C}$ is an exact category, then Condition (WIC) is equivalent to $\mathscr{C}$ is weakly idempotent complete (see [18] of [Proposition 7.6]).

(2) If $\mathscr{C}$ is a triangulated category, then Condition (WIC) is automatically satisfied.

Lemma 1. (c.f. [12] of [Proposition 3.15], [17]) Assume that $(\mathscr{C}, \mathbb{E}, \mathfrak{s})$ is an extriangulated category.

(1) Let $C$ be an object in $\mathscr{C}$, and let $A_{1} \stackrel{x_{1}}{\longrightarrow} B_{1} \stackrel{y_{1}}{\longrightarrow} C-\stackrel{\delta_{1}}{\rightarrow}$ and $A_{2} \stackrel{x_{2}}{\longrightarrow} B_{2} \stackrel{y_{2}}{\longrightarrow} C_{-}^{-\delta_{2}}>$ be any pair of $\mathbb{E}$-triangles. Then there is a commutative diagram in $\mathscr{C}$

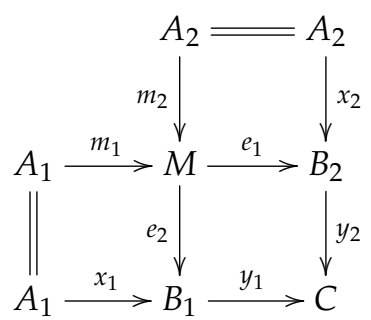

which satisfies $\mathfrak{s}\left(y_{2}^{*} \delta_{1}\right)=\left[A_{1} \stackrel{m_{1}}{\longrightarrow} M \stackrel{e_{1}}{\longrightarrow} B_{2}\right]$ and $\mathfrak{s}\left(y_{1}^{*} \delta_{2}\right)=\left[A_{2} \stackrel{m_{2}}{\longrightarrow} M \stackrel{e_{2}}{\longrightarrow} B_{1}\right]$.

(2) Let $A$ be an object in $\mathscr{C}$, and let $A \stackrel{x_{1}}{\longrightarrow} B_{1} \stackrel{y_{1}}{\longrightarrow} C_{1} \stackrel{\delta_{1}}{\longrightarrow}>$ and $A \stackrel{x_{2}}{\longrightarrow} B_{2} \stackrel{y_{2}}{\longrightarrow} C_{2}-{ }_{-}^{\delta_{2}} \rightarrow$ be any pair of $\mathbb{E}$-triangles. Then there is a commutative diagram in $\mathscr{C}$

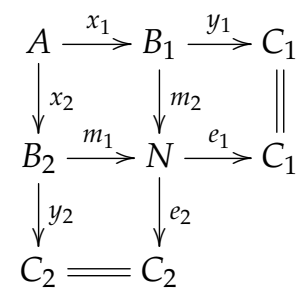

which satisfies $\mathfrak{s}\left(x_{2 *} \delta_{1}\right)=\left[B_{2} \stackrel{m_{1}}{\longrightarrow} N \stackrel{e_{1}}{\longrightarrow} C_{1}\right]$ and $\mathfrak{s}\left(x_{1 *} \delta_{2}\right)=\left[B_{1} \stackrel{m_{2}}{\longrightarrow} N \stackrel{e_{2}}{\longrightarrow} C_{2}\right]$.

The following definitions are quoted verbatim from [13] of [Section 3]. A class of $\mathbb{E}$-triangles $\xi$ is closed under base change if for any $\mathbb{E}$-triangle

$$
A \stackrel{x}{\longrightarrow} B \stackrel{y}{\longrightarrow} C-\stackrel{\delta}{-}>\in \xi
$$

and any morphism $c: C^{\prime} \rightarrow C$, then any $\mathbb{E}$-triangle $A \stackrel{x^{\prime}}{\rightarrow} B^{\prime} \stackrel{y^{\prime}}{\rightarrow} C^{\prime} \stackrel{c^{*} \delta}{\rightarrow}$ belongs to $\xi$.

Dually, a class of $\mathbb{E}$-triangles $\xi$ is closed under cobase change if for any $\mathbb{E}$-triangle

$$
A \stackrel{x}{\longrightarrow} B \stackrel{y}{\longrightarrow} C-\stackrel{\delta}{\rightarrow} \in \xi
$$

and any morphism $a: A \rightarrow A^{\prime}$, then any $\mathbb{E}$-triangle $A^{\prime} \stackrel{x^{\prime}}{\longrightarrow} B^{\prime} \stackrel{y^{\prime}}{\longrightarrow} C \stackrel{a_{*} \delta}{\rightarrow}$ belongs to $\xi$. A class of $\mathbb{E}$-triangles $\xi$ is called saturated if in the situation of Lemma 1(1), whenever $A_{2} \stackrel{x_{2}}{\longrightarrow} B_{2} \stackrel{y_{2}}{\longrightarrow} C_{-} \stackrel{\delta_{2}}{\rightarrow}$ and $A_{1} \stackrel{m_{1}}{\longrightarrow} M \stackrel{e_{1}}{\longrightarrow} B_{2} \stackrel{y_{2}^{*} \delta_{1}}{\longrightarrow}$ belong to $\xi$, then the $\mathbb{E}$-triangle

$$
A_{1} \stackrel{x_{1}}{\longrightarrow} B_{1} \stackrel{y_{1}}{\longrightarrow} C \stackrel{\delta_{1}}{-}>
$$


belongs to $\xi$.

An $\mathbb{E}$-triangle $A \stackrel{x}{\longrightarrow} B \stackrel{y}{\longrightarrow} C-\stackrel{\delta}{-}=$ is called split if $\delta=0$. It is easy to see that it is split if and only if $x$ is section or $y$ is retraction.

The full subcategory consisting of the split $\mathbb{E}$-triangles will be denoted by $\Delta_{0}$.

Definition 5. ([13] of [Definition 3.1], [17]) Let $\xi$ be a class of $\mathbb{E}$-triangles which is closed under isomorphisms. Then $\xi$ is called a proper class of $\mathbb{E}$-triangles if the following conditions hold:

(a) $\xi$ is closed under finite coproducts and $\Delta_{0} \subseteq \xi$.

(b) $\xi$ is closed under base change and cobase change.

(c) $\xi$ is saturated.

A proper class is a class which is not a set in general.

Definition 6. ([13] of [Definition 4.1], [17]) An object $P \in \mathscr{C}$ is called $\xi$-projective if for any $\mathbb{E}$-triangle

$$
A \stackrel{x}{\longrightarrow} B \stackrel{y}{\longrightarrow} C-\stackrel{\delta}{\rightarrow}
$$

in $\xi$, the induced sequence of abelian groups

$$
0 \longrightarrow \operatorname{Hom}_{\mathscr{C}}(P, A) \longrightarrow \operatorname{Hom}_{\mathscr{C}}(P, B) \longrightarrow \operatorname{Hom}_{\mathscr{C}}(P, C) \longrightarrow 0
$$

is exact. Dually, we have the definition of $\xi$-injective objects.

We denote by $\mathcal{P}(\xi)($ resp., $\mathcal{I}(\xi)$ ) the full subcategory of $\mathscr{C}$ consisting of $\xi$-projective (resp., $\xi$-injective) objects. It follows from the definition that $\mathcal{P}(\xi)$ and $\mathcal{I}(\xi)$ are full, additive, closed under isomorphisms and direct summands.

An extriangulated category $(\mathscr{C}, \mathbb{E}, \mathfrak{s})$ is said to have enough $\xi$-projectives (resp., enough $\xi$-injectives) provided that for each object $A$ there exists an $\mathbb{E}$-triangle $K \longrightarrow P \rightarrow A->$ (resp., $A \rightarrow I \rightarrow K->$ ) in $\xi$ with $P \in \mathcal{P}(\xi)$ (resp., $I \in \mathcal{I}(\xi)$ ).

The $\xi$-projective dimension $\xi$-pd $A$ of $A \in \mathscr{C}$ is defined inductively. If $A \in \mathcal{P}(\xi)$, then define $\xi$-pd $A=0$. For a positive integer $n$, one writes $\xi$-pd $A=n$ provided

(a) there is an $\mathbb{E}$-triangle $K \rightarrow P \rightarrow A->$ with $P \in \mathcal{P}(\xi)$ and $\xi$-pd $K=n-1$,

(b) there does not exist an $\mathbb{E}$-triangle $L \rightarrow P^{\prime} \rightarrow A->$ with $P^{\prime} \in \mathcal{P}(\xi)$ and $\xi$-pd $L<n-1$.

Of course we set $\xi-\operatorname{pd} A=\infty$, if $\xi-\operatorname{pd} A \neq n$ for all $n \geq 0$.

Dually we can define the $\xi$-injective dimension $\xi$-id $A$ of an object $A \in \mathscr{C}$.

Definition 7. ([13] of [Definition 4.4], [17]) $A \xi$-exact complex $\mathbf{X}$ is a diagram

$$
\cdots \longrightarrow X_{1} \stackrel{d_{1}}{\longrightarrow} X_{0} \stackrel{d_{0}}{\longrightarrow} X_{-1} \longrightarrow \cdots
$$

in $\mathscr{C}$ such that for each integer $n$, we have $d_{n}=g_{n-1} f_{n}$ for some $\mathbb{E}$-triangle

$$
K_{n+1} \stackrel{g_{n}}{\rightarrow} X_{n} \stackrel{f_{n}}{\longrightarrow} K_{n} \rightarrow
$$

in $\xi$.

In particular, by saying that

$$
X_{n} \stackrel{d_{n}}{\rightarrow} X_{n-1} \longrightarrow \cdots \rightarrow X_{1} \stackrel{d_{1}}{\rightarrow} X_{0}
$$

is $\xi$-exact, it means that there are $\mathbb{E}$-triangles

$$
X_{n} \stackrel{d_{n}}{\longrightarrow} X_{n-1} \stackrel{f_{n-1}}{\longrightarrow} K_{n-1}->\text { and } K_{2} \stackrel{g_{1}}{\longrightarrow} X_{1} \stackrel{d_{1}}{\longrightarrow} X_{0}->
$$


in $\xi$, and for each integer $1<i<n-1$, we have $d_{i}=g_{i-1} f_{i}$ for some $\mathbb{E}$-triangle

$$
K_{i+1} \stackrel{g_{i}}{\rightarrow} X_{i} \stackrel{f_{i}}{\rightarrow} K_{i}->
$$

in $\xi$.

Definition 8. ([8] of [Definition 3.1], [17]) Let $M$ be an object in $\mathscr{C}$. By a $\xi$-projective resolution of $M$ we mean a symbol of the form $\mathbf{P} \rightarrow M$ where $\mathbf{P}$ is a $\xi$-exact complex, where $P_{n} \in \mathcal{P}(\xi)$ for all $n \geq 0$ and where $P_{-1}=M$ and $P_{n}=0$ for all $n<-1$.

The notion of $\xi$-injective coresolution of $M$ is given dually.

Definition 9. ([8] of [Definition 3.2], [17]) Let $M$ and $N$ be objects in $\mathscr{C}$.

(1) If we choose a $\xi$-projective resolution $\mathbf{P} \longrightarrow M$ of $M$, by applying the functor $\mathscr{C}(-, N)$ to $\mathbf{P}$ we have a complex of abelian groups $\mathscr{C}(\mathbf{P}, N)$. For any integer $n \geq 0$, the $\xi$-cohomology groups $\xi \mathrm{xt}_{\mathcal{P}(\xi)}^{n}(M, N)$ are defined as

$$
\tilde{\xi} \mathbf{x t}_{\mathcal{P}(\xi)}^{n}(M, N)=H^{n}(\mathscr{C}(\mathbf{P}, N)) .
$$

(2) If we choose a $\tilde{\xi}$-injective coresolution $N \longrightarrow \mathbf{I}$ of $N$, by applying the functor $\mathscr{C}(M,-)$ to I we have a complex of abelian groups $\mathscr{C}(M, \mathbf{I})$. For any integer $n \geq 0$, the $\xi$-cohomology groups $\xi \mathbf{x t}_{\mathcal{I}(\xi)}^{n}(M, N)$ are defined as

$$
\tilde{\xi} \times \mathbf{t}_{\mathcal{I}(\xi)}^{n}(M, N)=H^{n}(\mathscr{C}(M, \mathbf{I}))
$$

Remark 3. (1) In fact, there is an isomorphism $\xi \times \mathbf{t}_{\mathcal{P}(\xi)}^{n}(M, N) \cong \xi \times \mathbf{x}_{\mathcal{I}(\xi)}^{n}(M, N)$, which is denoted by $\xi \mathrm{xt}_{\tilde{\xi}}^{n}(M, N)$ (see [8] of [Definition 3.2]).

(2) Assume that $\mathscr{C}$ has enough $\xi$-projective objects. Using a standard argument in homological algebra, there is a bijection

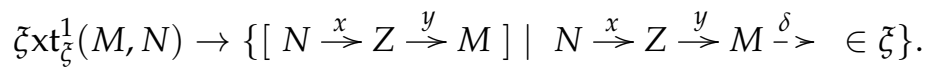

Remark 4. ([8] of [Lemma 3.4]) Let

$$
X \rightarrow Y \rightarrow Z->
$$

be an $\mathbb{E}$-triangle in $\xi$. If $\mathscr{C}$ has enough $\xi$-projective objects and $M$ is an object in $\mathscr{C}$, then there exists a long exact sequence

$$
\begin{aligned}
& 0 \longrightarrow \xi \mathrm{xt}_{\xi}^{0}(Z, M) \longrightarrow \xi \mathrm{xt}_{\tilde{\xi}}^{0}(Y, M) \longrightarrow \xi \mathrm{xt}_{\tilde{\xi}}^{0}(X, M) \longrightarrow \\
& \xi \operatorname{xt}_{\tilde{\xi}}^{1}(Z, M) \longrightarrow \mathcal{\xi}^{2} \mathrm{t}_{\tilde{\xi}}^{1}(Y, M) \longrightarrow \bar{\xi} \mathrm{xt}_{\tilde{\xi}}^{1}(X, M) \longrightarrow \cdots
\end{aligned}
$$

of abelian groups. If $\mathscr{C}$ has enough $\xi$-injective objects and $N$ is an object in $\mathscr{C}$, then there exists a long exact sequence

$$
\begin{aligned}
& 0 \longrightarrow \xi x \mathrm{t}_{\tilde{\xi}}^{0}(N, X) \longrightarrow \xi \mathrm{xt}_{\tilde{\xi}}^{0}(N, Y) \longrightarrow \xi x \mathrm{t}_{\tilde{\xi}}^{0}(N, Z) \longrightarrow \\
& \xi \mathrm{xt}_{\tilde{\xi}}^{1}(N, X) \longrightarrow \xi \mathrm{xt}_{\tilde{\xi}}^{1}(N, Y) \longrightarrow \xi \mathrm{xt}_{\tilde{\xi}}^{1}(N, Z) \rightarrow \cdots
\end{aligned}
$$

of abelian groups.

Now, we set

$$
\begin{aligned}
& \mathfrak{X}^{\perp}=\left\{M \in \mathscr{C} \mid \xi x t_{\tilde{\xi}}^{n \geq 1}(X, M)=0 \text { for all } X \in \mathfrak{X}\right\} \\
& { }^{\perp} \mathfrak{X}=\left\{M \in \mathscr{C} \mid \xi x t_{\tilde{\xi}}^{n \geq 1}(M, X)=0 \text { for all } X \in \mathfrak{X}\right\} .
\end{aligned}
$$


For two subcategories $\mathfrak{H}$ and $\mathfrak{X}$ of $\mathscr{C}$, we say $\mathfrak{H} \perp \mathfrak{X}$ if $\mathfrak{H} \subseteq \perp \mathfrak{X}$ (equivalently, $\mathfrak{X} \subseteq \mathfrak{H}^{\perp}$ ).

Definition 10. ([13] of [Definition 4.5], [17]) Let $\mathcal{W}$ be a class of objects in $\mathscr{C}$. An $\mathbb{E}$-triangle

$$
A \longrightarrow B \longrightarrow C_{-->}
$$

in $\xi$ is called to be $\mathscr{C}(-, \mathcal{W})$-exact (resp., $\mathscr{C}(\mathcal{W},-)$-exact) if for any $W \in \mathcal{W}$, the induced sequence of abelian groups $0 \longrightarrow \mathscr{C}(C, W) \longrightarrow \mathscr{C}(B, W) \longrightarrow \mathscr{C}(A, W) \longrightarrow 0$ (resp., $0 \longrightarrow \mathscr{C}(W, A) \longrightarrow \mathscr{C}(W, B) \longrightarrow \mathscr{C}(W, C) \longrightarrow 0)$ is exact in $\mathrm{Ab}$.

Definition 11. ([13] of [Definition 4.6], [17]) Let $\mathcal{W}$ be a class of objects in $\mathscr{C}$. A complex $\mathbf{X}$ is called $\mathscr{C}(-, \mathcal{W})$-exact (resp., $\mathscr{C}(\mathcal{W},-)$-exact) if it is a $\xi$-exact complex

$$
\cdots \longrightarrow X_{1} \stackrel{d_{1}}{\longrightarrow} X_{0} \stackrel{d_{0}}{\longrightarrow} X_{-1} \longrightarrow \cdots
$$

in $\mathscr{C}$ such that for each integer $n$ we have $d_{n}=g_{n-1} f_{n}$ for some $\mathscr{C}(-, \mathcal{W})$-exact $($ resp., $\mathscr{C}(\mathcal{W},-)$ exact) $\mathbb{E}$-triangle

$$
K_{n+1} \stackrel{g_{n}}{\longrightarrow} X_{n} \stackrel{f_{n}}{\longrightarrow} K_{n}-\stackrel{\delta_{n}}{-} \rightarrow
$$

in $\xi$.

A $\xi$-exact complex $\mathbf{X}$ is called complete $\mathcal{P}(\xi)$-exact (resp., complete $\mathcal{I}(\xi)$-exact) if it is $\mathscr{C}(-, \mathcal{P}(\xi))$-exact (resp., $\mathscr{C}(\mathcal{I}(\xi),-)$-exact).

Definition 12. ([13] of [Definition 4.7], [17]) A complete $\xi$-projective resolution is a complete $\mathcal{P}(\xi)$-exact complex

$$
\mathbf{P}: \cdots \longrightarrow P_{1} \stackrel{d_{1}}{\longrightarrow} P_{0} \stackrel{d_{0}}{\longrightarrow} P_{-1} \longrightarrow \cdots
$$

in $\mathscr{C}$ such that $P_{n}$ is $\xi$-projective for each integer $n$. Dually, a complete $\xi$-injective coresolution is a complete $\mathcal{I}(\xi)$-exact complex

$$
\mathbf{I}: \cdots \longrightarrow I_{1} \stackrel{d_{1}}{\longrightarrow} I_{0} \stackrel{d_{0}}{\longrightarrow} I_{-1} \longrightarrow \cdots
$$

in $\mathscr{C}$ such that $I_{n}$ is $\xi$-injective for each integer $n$.

Definition 13. ([13] of [Definition 4.8], [17]) Let $\mathbf{P}$ be a complete $\xi$-projective resolution in $\mathscr{C}$. Therefore, for each integer $n$, there exists a $\mathscr{C}(-, \mathcal{P}(\xi))$-exact $\mathbb{E}$-triangle

$$
K_{n+1} \stackrel{g_{n}}{\longrightarrow} P_{n} \stackrel{f_{n}}{\longrightarrow} K_{n}-\stackrel{\delta_{n}}{-}>
$$

in $\xi$. The objects $K_{n}$ are called $\xi$-Gprojective for each integer $n$.

Dually if $\mathbf{I}$ is a complete $\xi$-injective coresolution in $\mathscr{C}$, there exists a $\mathscr{C}(\mathcal{I}(\xi),-)$-exact E-triangle

$$
K_{n+1} \stackrel{g_{n}}{\longrightarrow} I_{n} \stackrel{f_{n}}{\longrightarrow} K_{n} \stackrel{\delta_{n}}{-}>
$$

in $\xi$ for each integer $n$. The objects $K_{n}$ are called $\xi$-Ginjective for each integer $n$.

We denote by $\mathcal{G P}(\xi)$ (resp., $\mathcal{G} \mathcal{I}(\xi)$ ) the class of $\xi$-Gprojective (resp., $\xi$-Ginjective) objects. It is obvious that $\mathcal{P}(\xi) \subseteq \mathcal{G} \mathcal{P}(\xi)$ and $\mathcal{I}(\xi) \subseteq \mathcal{G I}(\xi)$.

Definition 14. Let $\mathfrak{H}$ and $\mathfrak{X}$ be two subcategories of $\mathscr{C}$ with $\mathfrak{H} \subseteq \mathfrak{X}$. Then $\mathfrak{H}$ is called a $\mathfrak{\xi}$-cogenerator of $\mathfrak{X}$ if for any object $X$ in $\mathfrak{X}$, there exists an $\mathbb{E}$-triangle

$$
X \rightarrow H \rightarrow Z-\rightarrow
$$


in $\xi$ with $H \in \mathfrak{H}$ and $Z \in \mathfrak{X}$.

Definition 15. ([13] of [Definition 3.4]) Let

$$
X \stackrel{u}{\longrightarrow} Y \stackrel{v}{\longrightarrow} Z->
$$

be an $\mathbb{E}$-triangle in $\xi$. Then the morphism $u$ (resp. $v$ ) is called a $\xi$-infaltion (resp. a $\xi$-deflation).

Fix some arbitrary $\mathbb{E}$-triangle

$$
X \rightarrow Y \rightarrow Z->
$$

in $\xi$. We say that $\mathfrak{X}$ is closed under $\xi$-extensions if, given any such $\mathbb{E}$-triangle in $\xi$ as above, if $X, Z$ lie in $\mathfrak{X}$, then $Y$ lie in $\mathfrak{X}$. We say that $\mathfrak{X}$ is closed under cocones of $\xi$-deflations (resp. cones of $\xi$-inflations) if, given any such $\mathbb{E}$-triangle in $\xi$ as above, if $Y, Z$ lie in $\mathfrak{X}$ (resp. $X, Y$ lie in $\mathfrak{X})$, the so too does $X$ (resp. $Z$ ).

Definition 16. Let $\mathscr{C}$ be an extriangulated category with enough $\xi$-projective objects and $\mathfrak{X}$ a subcategory of $\mathscr{C}$. Then $\mathfrak{X}$ is called a resolving subcategory of $\mathscr{C}$ if the following conditions are satisfied.

(1) $\mathcal{P}(\xi) \subseteq \mathfrak{X}$.

(2) $\mathfrak{X}$ is closed under $\xi$-extensions.

(3) $\mathfrak{X}$ is closed under cocones of $\mathfrak{\xi}$-deflations.

Remark 5. (a) We do not require that a resolving subcategory is closed under direct summands in the above definition.

(b) $\mathcal{P}(\xi)$ is a resolving subcategory and closed under direct summands.

(c) $\mathcal{G} \mathcal{P}(\xi)$ is a resolving subcategory and closed under direct summands (see [13] of [Theorems 4.16 and 4.17]).

In the following sections, we always assume that $\mathscr{C}=(\mathscr{C}, \mathbb{E}, \mathfrak{s})$ is an extriangulated category and $\xi$ is a proper class of $\mathbb{E}$-triangles in $\mathscr{C}$. We also assume that the extriangulated category $\mathscr{C}$ has enough $\xi$-projectives and enough $\xi$-injectives satisfying Condition (WIC).

\section{Resolution Dimension with Respect to a Resolving Subcategory}

We first introduce the following definition.

Definition 17. Let $\mathfrak{X}$ be a subcategory of $\mathscr{C}$ and $M \in \mathscr{C}$. The $\mathfrak{X}$-resolution dimension of $M$ (with respect to $\xi$ ), written $\mathfrak{X}$-res.dim $M$, is defined by

$\mathfrak{X}$-res.dim $M=\inf \{n \geq 0 \mid$ there exists a $\tilde{\xi}$-exact complex

$$
\left.X_{n} \rightarrow \cdots \rightarrow X_{1} \rightarrow \longrightarrow X_{0} \rightarrow M \text { in } \mathscr{C} \text { with all } X_{i} \text { objects in } \mathfrak{X}\right\} .
$$

For a $\xi$-exact complex

$$
\cdots \stackrel{f_{n+1}}{\longrightarrow} X_{n} \longrightarrow \cdots \stackrel{f_{2}}{\longrightarrow} X_{1} \stackrel{f_{1}}{\longrightarrow} X_{0} \stackrel{f_{0}}{\longrightarrow} M
$$

with all $X_{i} \in \mathfrak{X}$, there are $\mathbb{E}$-triangles $K_{1} \stackrel{g_{0}}{\longrightarrow} X_{0} \stackrel{f_{0}}{\rightarrow} M->$ and $K_{i+1} \stackrel{g_{i}}{\rightarrow} X_{i} \stackrel{h_{i}}{\rightarrow} K_{i}->$ with $f_{i}=g_{i-1} h_{i}$ for each $i>0$. The object $K_{i}$ are called an ith $\mathfrak{X}$-syzygy of $M$, denoted by $\Omega_{\mathfrak{X}}^{i}(M)$. In case $\mathfrak{X}=\mathcal{P}(\mathfrak{\xi})$, we have $\xi_{\text {- }}$ pd $M=\mathfrak{X}$-res.dim $M$ and write $\Omega^{i}(M):=\Omega_{\mathcal{P}(\xi)}^{i}(M)$. In case $\mathfrak{X}=\mathcal{G} \mathcal{P}(\xi), \mathfrak{X}$-res.dim $M$ coincides with $\xi-\mathcal{G}$ pd $M$ defined by Hu, Zhang and Zhou [13] as $\xi$-G projective dimension, the proof is straightforward. 
Lemma 2. Let $\mathfrak{X}$ be a resolving subcategory of $\mathscr{C}$. For any object $M \in \mathscr{C}$, if

$$
X_{n} \stackrel{f_{n}}{\rightarrow} \cdots \rightarrow X_{1} \stackrel{f_{1}}{\rightarrow} X_{0} \stackrel{f_{0}}{\longrightarrow} M
$$

and

$$
Y_{n} \stackrel{g_{n}}{\longrightarrow} \cdots \longrightarrow Y_{1} \stackrel{g_{1}}{\longrightarrow} Y_{0} \stackrel{g_{0}}{\longrightarrow} M
$$

are $\mathfrak{\xi}$-exact complexes with all $X_{i}$ and $Y_{i}$ in $\mathfrak{X}$ for $0 \leq i \leq n-1$, then $X_{n} \in \mathfrak{X}$ if and only if $Y_{n} \in \mathfrak{X}$.

Proof. For $M \in \mathscr{C}$, since $\mathscr{C}$ has enough $\xi$-projectives, there exists a $\xi$-exact complex

$$
K_{n} \stackrel{u_{n}}{\longrightarrow} P_{n-1} \stackrel{h_{n-1}}{\longrightarrow} \cdots \rightarrow P_{1} \stackrel{h_{1}}{\longrightarrow} P_{0} \stackrel{h_{0}}{\longrightarrow} M
$$

with $P_{i} \in \mathcal{P}(\xi)$ for $0 \leq i \leq n-1$.

First of all, by the $\xi$-exact complex (1) there are $\mathbb{E}$-triangles

$$
K_{1}^{M} \stackrel{t_{1}}{\longrightarrow} X_{0} \stackrel{f_{0}}{\longrightarrow} M->\text { and } K_{2}^{M} \stackrel{t_{2}}{\longrightarrow} X_{1} \stackrel{s_{1}}{\rightarrow} K_{1}^{M}->
$$

in $\xi$ with $f_{1}=t_{1} s_{1}$. Moreover, by the $\xi$-exact complex (3) there is an $\mathbb{E}$-triangle $K_{1} \stackrel{u_{1}}{\longrightarrow} P_{0} \stackrel{h_{0}}{\longrightarrow} M->$ in $\xi$. Consider the following diagram

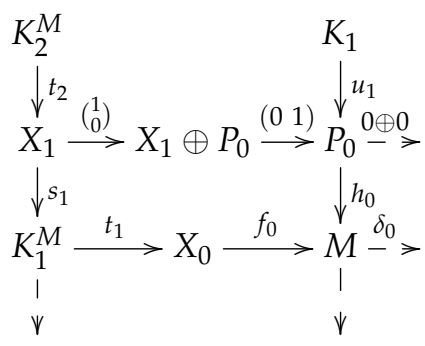

It is easy to see that $s_{1 *}(0 \oplus 0)=0=h_{0}{ }^{*}\left(\delta_{0}\right)$, i.e., $\left(s_{1}, h_{0}\right): 0 \oplus 0 \rightarrow \delta_{0}$ is a morphism of E-extensions. Thus, by [13] of [Lemma 4.15], there is an E-triangle $\overline{K_{1}} \stackrel{w_{1}}{\longrightarrow} X_{1} \oplus P_{0} \stackrel{q_{1}}{\longrightarrow} X_{0}->$ such that the following diagram

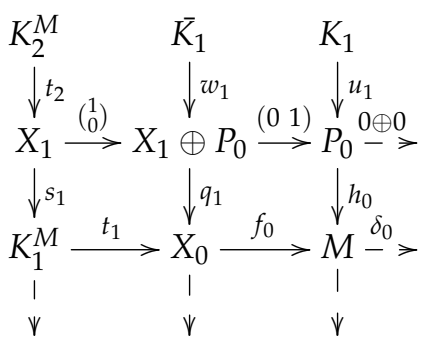


commutes. By [13] of [Lemma 4.14], there exist morphisms $k_{2}: K_{2}^{M} \rightarrow \overline{K_{1}}$ and $k_{1}$ : $\overline{K_{1}} \rightarrow K_{1}$ such that there is an $\mathbb{E}$-triangle $K_{2}^{M} \stackrel{k_{2}}{\rightarrow} \overline{K_{1}} \stackrel{k_{1}}{\rightarrow} K_{1}->$ and meanwhile, the following diagram

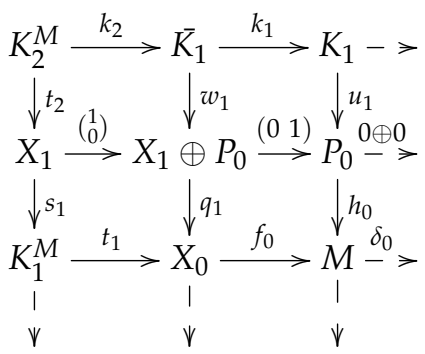

commutes. Repeating this process, we can obtain the following $\xi$-exact complex

$$
K_{n} \stackrel{w_{n}}{\longrightarrow} X_{n} \oplus P_{n-1} \longrightarrow X_{n-1} \oplus P_{n-2} \longrightarrow \cdots \longrightarrow X_{2} \oplus P_{1} \longrightarrow X_{1} \oplus P_{0} \stackrel{q_{1}}{\longrightarrow} X_{0} .
$$

Similarly, we have the following $\xi$-exact complex

$$
K_{n} \stackrel{z_{n}}{\longrightarrow} Y_{n} \oplus P_{n-1} \longrightarrow Y_{n-1} \oplus P_{n-2} \longrightarrow \cdots \longrightarrow Y_{2} \oplus P_{1} \longrightarrow Y_{1} \oplus P_{0} \stackrel{p_{1}}{\longrightarrow} Y_{0} .
$$

Decompose the $\xi$-exact complex (4) as the $\mathbb{E}$-triangle

$$
K_{n} \stackrel{w_{n}}{\rightarrow} X_{n} \oplus P_{n-1} \longrightarrow X->
$$

in $\xi$ and the $\xi$-exact complex

$$
X \rightarrow X_{n-1} \oplus P_{n-2} \longrightarrow \cdots \rightarrow X_{2} \oplus P_{1} \rightarrow X_{1} \oplus P_{0} \stackrel{q_{1}}{\longrightarrow} X_{0} .
$$

Decompose the $\xi$-exact complex (5) as the $\mathbb{E}$-triangle

$$
K_{n} \stackrel{z_{n}}{\longrightarrow} Y_{n} \oplus P_{n-1} \longrightarrow Y_{->}
$$

in $\xi$ and the $\xi$-exact complex

$$
Y \rightarrow Y_{n-1} \oplus P_{n-2} \longrightarrow \cdots \rightarrow Y_{2} \oplus P_{1} \longrightarrow Y_{1} \oplus P_{0} \stackrel{p_{1}}{\rightarrow} Y_{0} .
$$

Since $\mathfrak{X}$ is resolving, we have that $X$ and $Y$ are objects in $\mathfrak{X}$ by $\mathcal{\xi}$-exact complexes (7) and (9). Moreover, by $\mathbb{E}$-triangles (6) and (8) we have that $X_{n} \oplus P_{n-1} \in \mathfrak{X}$ if and only if $K_{n} \in \mathfrak{X}$ if and only if $Y_{n} \oplus P_{n-1} \in \mathfrak{X}$.

However, from the following $\mathbb{E}$-triangles in $\xi$

$$
X_{n} \stackrel{\left(\begin{array}{l}
1 \\
0
\end{array}\right)}{\longrightarrow} X_{n} \oplus P_{n-1} \stackrel{\left(\begin{array}{ll}
0 & 1
\end{array}\right)}{\longrightarrow} P_{n-1} \stackrel{0}{\rightarrow} \text { and } Y_{n} \stackrel{\left(\begin{array}{l}
1 \\
0
\end{array}\right)}{\longrightarrow} Y_{n} \oplus P_{n-1} \stackrel{\left(\begin{array}{ll}
0 & 1
\end{array}\right)}{\longrightarrow} P_{n-1} \stackrel{0}{\rightarrow},
$$

we have that $X_{n} \in \mathfrak{X}$ if and only if $X_{n} \oplus P_{n-1} \in \mathfrak{X}$, and $Y_{n} \in \mathfrak{X}$ if and only if $Y_{n} \oplus P_{n-1} \in \mathfrak{X}$. Thus, $X_{n} \in \mathfrak{X}$ if and only if $Y_{n} \in \mathfrak{X}$.

Using the above, we can get

Proposition 1. Let $\mathfrak{X}$ be a resolving subcategory of $\mathscr{C}$ and $M \in \mathscr{C}$. Then the following statements are equivalent:

(1) $\mathfrak{X}$-res.dim $M \leq m$.

(2) $\Omega^{n}(M) \in \mathfrak{X}$ for $n \geq m$.

(3) $\Omega_{\mathfrak{X}}^{n}(M) \in \mathfrak{X}$ for $n \geq m$. 
Proof. Apply Lemma 2.

Now we can compare resolution dimensions in a given $\mathbb{E}$-triangle in $\xi$ as follows.

Proposition 2. labelprop-resdim Let $\mathfrak{X}$ be a resolving subcategory of $\mathscr{C}$, and let

$$
A \longrightarrow B \longrightarrow C->
$$

be an $\mathbb{E}$-triangle in $\xi$. Then we have the following statements for any objects $A, B$ and $C$ in $\mathscr{C}$ :

(1) $\mathfrak{X}$-res.dim $B \leq \max \{\mathfrak{X}$-res.dim $A, \mathfrak{X}$-res.dim $C\}$.

(2) $\mathfrak{X}$-res.dim $A \leq \max \{\mathfrak{X}$-res.dim $B, \mathfrak{X}$-res.dim $C-1\}$.

(3) $\mathfrak{X}$-res.dim $C \leq \max \{\mathfrak{X}$-res.dim $A+1, \mathfrak{X}$-res.dim $B\}$.

Proof. For any $D \in \mathscr{C}$, if $\mathfrak{X}$-res.dim $D=d<\infty$, by Proposition 1 , we have the following $\xi$-exact complex

$$
P_{d}^{D} \longrightarrow P_{d-1}^{D} \rightarrow \cdots \rightarrow P_{1}^{D} \rightarrow P_{0}^{D} \rightarrow D
$$

in $\mathscr{C}$ with $P_{i}^{D} \in \mathcal{P}(\xi)$ for $0 \leq i \leq d-1$ and $P_{d}^{D} \in \mathfrak{X}$.

(1) Assume $\mathfrak{X}$-res.dim $A=m<\infty$ and $\mathfrak{X}$-res.dim $C=n<\infty$, We will use induction on $m$ and $n$. The case $m=n=0$ is trivial. Without loss of generality, we assume $m \leq n$, then we can let $P_{i}^{A}=0$ for $i>m$. As a similar argument to proof of Lemma 2, we can obtain the following $\xi$-exact complex

$$
P_{n}^{A} \oplus P_{n}^{C} \longrightarrow P_{n-1}^{A} \oplus P_{n-1}^{C} \longrightarrow \cdots \rightarrow P_{0}^{A} \oplus P_{0}^{C} \longrightarrow B
$$

in $\mathscr{C}$.

Thus, $\mathfrak{X}$-res.dim $B \leq n=\max \{\mathfrak{X}$-res.dim $A, \mathfrak{X}$-res.dim $C\}$.

(2) Assume $\mathfrak{X}$-res.dim $B=m<\infty$ and $\mathfrak{X}$-res.dim $C=n<\infty$. We will use induction on $m$ and $n$. The case $m=n=0$ is trivial. Without loss of generality, we assume $m \leq n-1$, then we can let $P_{i}^{B}=0$ for $i>m$. By [14] of [Theorem 1], there exist a $\xi$-exact complex

$$
P_{n}^{C} \oplus P_{n-1}^{B} \rightarrow P_{n-1}^{C} \oplus P_{n-2}^{B} \rightarrow \cdots \rightarrow P_{2}^{C} \oplus P_{1}^{B} \rightarrow K \rightarrow A
$$

and an $\mathbb{E}$-triangle

$$
K \longrightarrow P_{1}^{C} \oplus P_{0}^{B} \rightarrow P_{0}^{C}->
$$

in $\xi$, it follows that $K \in \mathcal{P}(\xi)$ by Remark 5. Thus, $\mathfrak{X}$-res.dim $A \leq n-1$ and the desired assertion is obtained.

(3) Assume $\mathfrak{X}$-res.dim $A=m<\infty$ and $\mathfrak{X}$-res.dim $B=n<\infty$. We proceed it by induction on $m$ and $n$. The case $m=n=0$ is trivial. Without loss of generality, we assume $m+1 \leq n$, then we can let $P_{i}^{A}=0$ for $i>m$. By [14] of [Theorem 3], we have the following $\xi$-exact complex

$$
P_{n}^{B} \oplus P_{n-1}^{A} \longrightarrow \cdots \rightarrow P_{2}^{B} \oplus P_{1}^{A} \longrightarrow P_{1}^{B} \oplus P_{0}^{A} \longrightarrow P_{0}^{B} \rightarrow C
$$

in $\mathscr{C}$, thus $\mathfrak{X}$-res.dim $A \leq n$ and the desired assertion is obtained.

We use $\widehat{\mathcal{X}}$ to denote the full subcategory of $\mathscr{C}$ whose objects have finite $\mathfrak{X}$-resolution dimension. Following the above, we have the closure properties for the subcategory $\widehat{\mathfrak{X}}$.

Remark 6. If $\mathfrak{X}$ is a resolving subcategory of $\mathscr{C}$, then $\widehat{\mathfrak{X}}$ is closed under cocones of $\mathfrak{\xi}$-deflations, cones of $\xi$-inflations and $\xi$-extensions. 
Corollary 1 . Let $\mathfrak{X}$ be a resolving subcategory of $\mathscr{C}$, and let

$$
A \rightarrow B \longrightarrow C->
$$

be an $\mathbb{E}$-triangle in $\xi$.

(1) Let $C \in \mathfrak{X}$. Then $\mathfrak{X}$-res.dim $A=\mathfrak{X}$-res.dim $B$.

(2) Let $B \in \mathfrak{X}$. Then either $A \in \mathfrak{X}$ or else $\mathfrak{X}$-res.dim $A=\mathfrak{X}$-res.dim $C-1$.

(3) Let $A \in \mathfrak{X}$ and $B, C \notin \mathfrak{X}$. Then $\mathfrak{X}$-res.dim $B=\mathfrak{X}$-res.dim $C$.

Proposition 3. Let $\mathfrak{H}$ and $\mathfrak{X}$ be two subcategories of $\mathscr{C}$ with $\mathfrak{H} \subseteq \mathfrak{X}$.

(1) $\widehat{\mathfrak{H}} \subseteq \widehat{\mathfrak{X}}$.

(2) If $\mathfrak{X}$ is resolving, then for any $M \in \widehat{\mathfrak{H}}$, $\mathfrak{H}$-res.dim $M=\mathfrak{X}$-res.dim $M$ if and only if $\widehat{\mathfrak{H}} \cap \mathfrak{X}=\mathfrak{H}$.

In particular, if $\mathfrak{X} \perp \mathfrak{H}$, and $\mathfrak{H}$ is closed under cocones of $\xi$-deflations or closed under direct summands, then $\widehat{\mathfrak{H}} \cap \mathfrak{X}=\mathfrak{H}$.

Proof. (1) Obviously.

(2) The only if part. Clearly, $\mathfrak{H} \subseteq \widehat{\mathfrak{H}} \cap \mathfrak{X}$. Let $M \in \widehat{\mathfrak{H}} \cap \mathfrak{X}$. By assumption, we have $\mathfrak{H}$-res.dim $M=\mathfrak{X}$-res.dim $M=0$, then $M \in \mathfrak{H}$, and so $\widehat{\mathfrak{H}} \cap \mathfrak{X} \subseteq \mathfrak{H}$. Thus, $\widehat{\mathfrak{H}} \cap \mathfrak{X}=\mathfrak{H}$.

The if part. Suppose $\mathfrak{H}$-res.dim $M=n<\infty$ and $\mathfrak{X}$-res.dim $M=m<\infty$. Clearly $m \leq n$. Consider the following $\xi$-exact complexes

$$
H_{n} \longrightarrow \cdots \rightarrow H_{0} \longrightarrow M
$$

and

$$
X_{m} \longrightarrow \cdots \rightarrow X_{0} \rightarrow M
$$

with $H_{i} \in \mathfrak{H}$ and $X_{j} \in \mathfrak{X}$ for all $0 \leq i \leq n$ and $0 \leq j \leq m$. Since $\mathfrak{H} \subseteq \mathfrak{X}$, we have $\Omega_{\mathfrak{H}}^{m}(M) \in \mathfrak{X}$ by Lemma 2 . Then $\Omega_{\mathfrak{H}}^{m}(M) \in \widehat{\mathfrak{H}} \cap \mathfrak{X}=\mathfrak{H}$, and thus $\mathfrak{H}$-res.dim $M \leq m$ and the desired equality is obtained.

Now, we assume that $\mathfrak{X} \perp \mathfrak{H}$ and $\mathfrak{H}$ is closed under cocones of $\xi$-deflations or closed under direct summands. Clearly, $\mathfrak{H} \subseteq \widehat{\mathfrak{H}} \cap \mathfrak{X}$. Conversely, let $M \in \widehat{\mathfrak{H}} \cap \mathfrak{X}$. There exists a $\xi$-exact complex

$$
H_{n} \longrightarrow H_{n-1} \longrightarrow \cdots \rightarrow H_{0} \longrightarrow M
$$

with each $H_{i}$ lies in $\mathfrak{H}$. Set $K_{i}=\operatorname{Cocone}\left(H_{i} \rightarrow H_{i-1}\right)$ for $0 \leq i \leq n-2$, where $H_{-1}=M$. Since $\mathfrak{X}$ is resolving, we have $K_{i} \in \mathfrak{X}$, and hence $K_{i} \in \widehat{\mathfrak{H}} \cap \mathfrak{X}$. Consider the following $\mathbb{E}$-triangle

$$
H_{n} \longrightarrow H_{n-1} \longrightarrow K_{n-2}->
$$

in $\xi$. Since $\xi x t_{\tilde{\xi}}^{1}\left(K_{n-2}, H_{n}\right)=0$ by the assumption that $\mathfrak{X} \perp \mathfrak{H}$, we have that the $\mathbb{E}$ triangle (10) is split by Remark 3(2). It follows that $H_{n-1} \cong H_{n} \oplus K_{n-2}$ and there exists an $\mathbb{E}$-triangle

$$
K_{n-2} \longrightarrow H_{n-1} \longrightarrow H_{n}{ }^{0}>
$$

in $\xi$. Since $\mathfrak{H}$ is closed under cocones of $\xi$-deflations or closed under direct summands by assumption, we have $K_{n-2} \in \mathfrak{H}$. Repeating this process, we can obtain each $K_{i} \in \mathfrak{H}$, hence $M \in \mathfrak{H}$ and $\widehat{\mathfrak{H}} \cap \mathfrak{X} \subseteq \mathfrak{H}$. Thus, $\widehat{\mathfrak{H}} \cap \mathfrak{X}=\mathfrak{H}$.

Now we give the following definition.

Definition 18. Let $\mathfrak{X}$ be a subcategory of $\mathscr{C}$ and $M$ an object in $\mathscr{C}$. A $\xi$-deflation $X \rightarrow M$ with $X \in \mathfrak{X}$ is said to be a right $\mathfrak{X}$-approximation of $M$ if $\operatorname{Hom}_{\mathscr{C}}(\widetilde{X}, X) \rightarrow \operatorname{Hom}_{\mathscr{C}}(\widetilde{X}, M) \rightarrow 0$ is exact for any $\widetilde{X} \in \mathfrak{X}$. 
The notion of a left $\mathfrak{X}$-approximation of $M$ is given dually.

We need the following easy and useful observation.

Lemma 3. Let $\mathfrak{H}$ and $\mathfrak{X}$ be two subcategories of $\mathscr{C}$.

(1) If $\mathfrak{X} \perp \mathfrak{H}$, then $\mathfrak{X} \perp \widehat{\mathfrak{H}}$. In particular, if $\mathfrak{H} \perp \mathfrak{H}$, then $\mathfrak{H} \perp \widehat{\mathfrak{H}}$.

(2) If $M \in{ }^{\perp} \mathfrak{H}$, then $M \in{ }^{\perp} \widehat{\mathfrak{H}}$.

Proof. let $M \in \widehat{\mathfrak{H}}$. Then there is a $\tilde{\xi}$-exact complex

$$
H_{n} \stackrel{h_{n}}{\longrightarrow} H_{n-1} \stackrel{h_{n-1}}{\longrightarrow} \cdots \rightarrow H_{0} \stackrel{h_{0}}{\longrightarrow} M
$$

with each $H_{i} \in \mathfrak{H}$ for some nonnegative integer $n$. This means that there are $\mathbb{E}$-triangles $H_{n} \stackrel{h_{n}}{\longrightarrow} H_{n-1} \stackrel{g_{n-1}}{\longrightarrow} K_{n-1}->, K_{1} \stackrel{w_{0}}{\longrightarrow} H_{0} \stackrel{h_{0}}{\longrightarrow} M->$, and $K_{i+1} \stackrel{w_{i}}{\longrightarrow} H_{i} \stackrel{g_{i}}{\longrightarrow} K_{i}->$ in $\xi$ with $h_{i}=w_{i-1} g_{i}$ for any $0<i<n-1$. Applying Remark 4, we can get $\xi \mathbf{x t}_{\xi}^{i}(X, M) \cong$ $\xi \mathrm{xt}_{\tilde{\xi}}^{i+1}\left(X, K_{1}\right) \cong \ldots \cong \xi \mathrm{xt}_{\tilde{\xi}}^{i+n}\left(X, H_{n}\right)=0$ for any $X \in \mathfrak{X}$.

The following is an analogous theory of Auslander-Buchweitz approximations (see $[3,6])$.

Proposition 4. Let $\mathfrak{X}$ be a subcategory of $\mathscr{C}$ closed under $\tilde{\xi}$-extensions, and let $\mathfrak{H}$ be a subcategory of $\mathfrak{X}$ such that $\mathfrak{H}$ is a $\mathfrak{\xi}$-cogenerator of $\mathfrak{X}$. Then for each $M \in \mathscr{C}$ with $\mathfrak{X}$-res.dim $M=n<\infty$, there exist two $\mathbb{E}$-triangles

$$
K \stackrel{f}{\rightarrow} X \stackrel{g}{\rightarrow} M->
$$

and

$$
M \stackrel{u}{\longrightarrow} W \stackrel{v}{\longrightarrow} X^{\prime}->
$$

in $\xi$, where $X, X^{\prime} \in \mathfrak{X}, \mathfrak{H}$-res.dim $K \leq n-1$ and $\mathfrak{H}$-res.dim $W \leq n$.

In particular, if $\mathfrak{X} \perp \mathfrak{H}$, then the $\xi$-deflation $g: X \rightarrow M$ is a right $\mathfrak{X}$-approximation of $M$, and the $\xi$-inflation $u: M \rightarrow W$ is a left $\widehat{\mathfrak{H}}$-approximation of $M$.

Proof. We will use induction on $n$. The case for $n=0$ is trivial. If $n=1$, there exists an $\mathbb{E}$-triangle

$$
X_{1} \rightarrow X_{0} \rightarrow M->
$$

in $\xi$ with $X_{0}, X_{1} \in \mathfrak{X}$. Since $\mathfrak{H}$ is a $\xi$-cogenerator of $\mathfrak{X}$, there is an $\mathbb{E}$-triangle

$$
X_{1} \rightarrow H \rightarrow X_{1}^{\prime} \rightarrow
$$

in $\xi$ with $H \in \mathfrak{H}$ and $X_{1}^{\prime} \in \mathfrak{X}$. By Lemma $1(2)$, we have the following commutative diagram

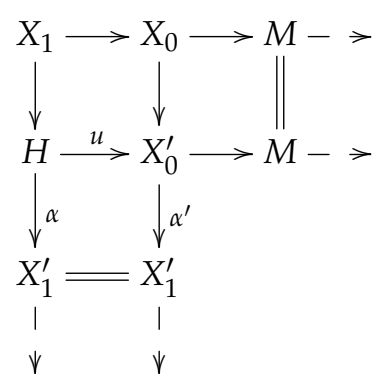


Since $\xi$ is closed under cobase changes, we obtain that the $\mathbb{E}$-triangle

$$
H \stackrel{u}{\rightarrow} X_{0}^{\prime} \longrightarrow M->
$$

is in $\xi$ with $\mathfrak{H}$-res.dim $H=0$. Notice that $\alpha^{\prime} u=\alpha$ is a $\xi$-deflation, so we have that $\alpha^{\prime}$ is a $\xi$-deflation by [13] of [Proposition 4.13], hence the $\mathbb{E}$-triangle

$$
X_{0} \rightarrow X_{0}^{\prime} \stackrel{\alpha^{\prime}}{\rightarrow} X_{1}^{\prime}->
$$

is in $\xi$ by [12] of [Remark 3.10]. Since $\mathfrak{X}$ is closed under $\xi$-extensions by assumption, we have $X_{0}^{\prime} \in \mathfrak{X}$. Therefore, (14) is the first desired $\mathbb{E}$-triangle.

For $X_{0}^{\prime}$, since $\mathfrak{H}$ is a $\mathfrak{\xi}$-cogenerator of $\mathfrak{X}$, there is an $\mathbb{E}$-triangle

$$
X_{0}^{\prime} \rightarrow H_{0} \rightarrow X_{0}^{\prime \prime}-\rightarrow
$$

in $\xi$ with $H_{0} \in \mathfrak{H}$ and $X_{0}^{\prime \prime} \in \mathfrak{X}$. By (ET4), we have the following commutative diagram

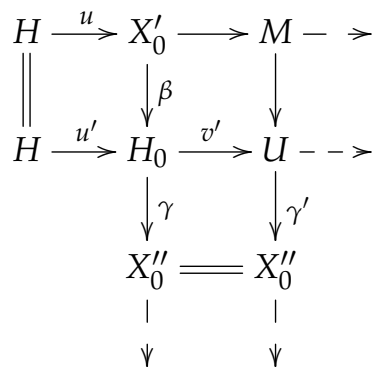

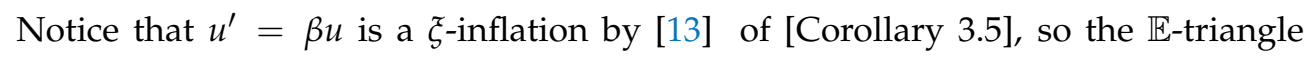
$H \stackrel{u^{\prime}}{\longrightarrow} H_{0} \stackrel{v^{\prime}}{\longrightarrow} U-\rightarrow$ is in $\xi$. Since $\gamma^{\prime} v^{\prime}=\gamma$ is a $\xi$-deflation, $\gamma^{\prime}$ is a $\xi$-deflation by [13] of [Proposition 4.13]. Therefore, the $\mathbb{E}$-triangle

$$
M \longrightarrow U \stackrel{\gamma^{\prime}}{\longrightarrow} X_{0}^{\prime \prime}->
$$

is in $\xi$ with $\mathfrak{H}$-res.dim $U \leq 1$ and $X_{0}^{\prime \prime} \in \mathfrak{X}$, which is the second desired $\mathbb{E}$-triangle.

Now suppose $n \geq 2$. Then there is an $\mathbb{E}$-triangle

$$
K^{\prime} \longrightarrow X_{0} \longrightarrow M->
$$

in $\xi$ with $\mathfrak{X}$-res.dim $K^{\prime} \leq n-1$ and $X_{0} \in \mathfrak{X}$. For $K^{\prime}$, by the induction hypothesis, we get an $\mathbb{E}$-triangle

$$
K^{\prime} \longrightarrow K \longrightarrow X_{2}->
$$

in $\xi$ with $\mathfrak{H}$-res.dim $K \leq n-1$ and $X_{2} \in \mathfrak{X}$. By Lemma 1(2), we have the following commutative diagram

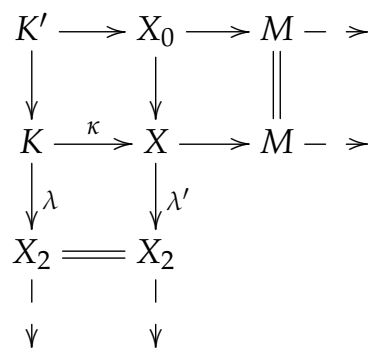


Notice that $\lambda^{\prime} \kappa=\lambda$ is a $\xi$-deflation, then $\lambda^{\prime}$ is a $\xi$-deflation by [13] of [Proposition 4.13], so the $\mathbb{E}$-triangle

$$
X_{0} \rightarrow X \rightarrow X_{2}->
$$

is in $\xi$. It follows that $X \in \mathfrak{X}$ from the assumption that $\mathfrak{X}$ is closed under $\mathfrak{\xi}$-extensions. Since $\xi$ is closed under cobase changes, we obtain the first desired $\mathbb{E}$-triangle

$$
K \longrightarrow X \rightarrow M->
$$

in $\tilde{\xi}$ with $\mathfrak{H}$-res.dim $K \leq n-1$ and $X \in \mathfrak{X}$.

For $X$, since $\mathfrak{H}$ is a $\mathfrak{\xi}$-cogenerator of $\mathfrak{X}$, we get the following $\mathbb{E}$-triangle

$$
X \rightarrow H_{1} \longrightarrow X^{\prime}->
$$

in $\xi$ with $H_{1} \in \mathfrak{H}$ and $X^{\prime} \in \mathfrak{X}$.

By (ET4), we have the following commutative diagram

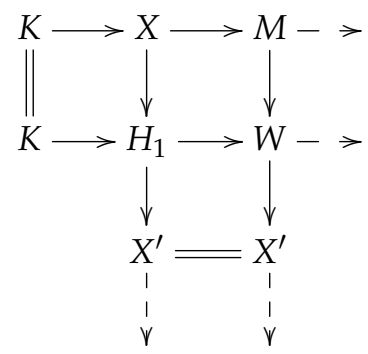

As a similar argument to that of the diagram (15), we obtain that the $\mathbb{E}$-triangles

$$
K \longrightarrow H_{1} \longrightarrow W->
$$

and

$$
M \longrightarrow W \longrightarrow X^{\prime}->
$$

are in $\xi$. Thus, (18) is the second desired $\mathbb{E}$-triangle in $\xi$ with $\mathfrak{H}$-res.dim $W \leq n$ and $X^{\prime} \in \mathfrak{X}$.

In particular, suppose $\mathfrak{X} \perp \mathfrak{H}$. By Lemma 3 , we have $\mathfrak{X} \perp \widehat{\mathfrak{H}}$. Then $\tilde{\xi} x t_{\widetilde{\xi}}^{1}(\widetilde{X}, K)=0$ for any $\widetilde{X} \in \mathfrak{X}$, it follows that $\operatorname{Hom}_{\mathscr{C}}(\widetilde{X}, X) \rightarrow \operatorname{Hom}_{\mathscr{C}}(\widetilde{X}, M) \rightarrow 0$ is exact. Thus, the $\xi$-deflation $X \rightarrow M$ is a right $\mathfrak{X}$-approximation of $M$. Similarly, we can prove that the $\xi$-inflation $u: M \rightarrow W$ is a left $\widehat{\mathfrak{H}}$-approximation of $M$.

Proposition 5. Keep the notion as Proposition 4. Assume $M \in \widehat{\mathfrak{X}}$ with $\mathfrak{X}$-res.dim $M=n<\infty$.

(1) If $\mathfrak{X}$ is resolving, then in the $\mathbb{E}$-triangles (11) and (12), we have $\mathfrak{H}$-res.dim $K=n-1$ and $\mathfrak{H}$-res.dim $W=\mathfrak{X}$ - res.dim $W=n$.

In particular, if $\mathfrak{X} \perp \mathfrak{H}$, then the $\xi$-deflation $X \rightarrow M$ in the $\mathbb{E}$-triangle (11) is a right $\mathfrak{X}$-approximation of $M$, such that $\mathfrak{H}$ - res.dim $K=n-1$.

(2) If $\mathfrak{X} \perp \mathfrak{H}$ and $\mathfrak{X}$ is resolving, then there is an $\mathbb{E}$-triangle

$$
M \longrightarrow M^{\prime} \longrightarrow X->
$$

in $\xi$ with $M^{\prime} \in \mathfrak{X}^{\perp}, X \in \mathfrak{X}$ and $\mathfrak{X}$-res.dim $M=\mathfrak{X}$-res.dim $M^{\prime}$.

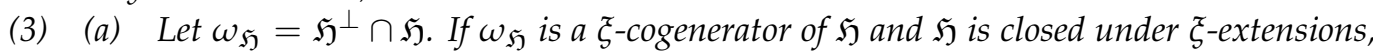
then $\mathfrak{X} \perp \omega_{\mathfrak{H}}$ if and only if $\mathfrak{X} \perp\left(\mathfrak{H}^{\perp} \cap \widehat{\mathfrak{H}}\right)$.

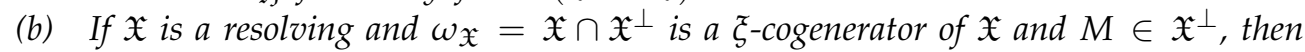
$\mathfrak{X}$-res.dim $M=\omega_{\mathfrak{X}}$-res.dim $M$. 


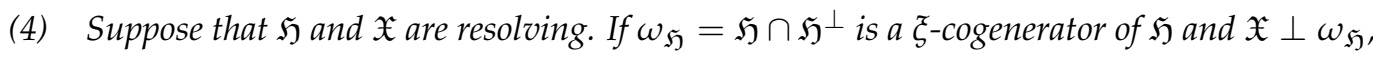
then $M$ admits a right $\mathfrak{X}$-approximation $X^{\prime} \rightarrow M$ such that $K^{\prime \prime} \rightarrow X^{\prime} \rightarrow M-\rightarrow$ is an $\mathbb{E}$-triangle in $\xi$, where $\mathfrak{H}$-res.dim $K^{\prime \prime}=n-1$. In fact, we have $\omega_{\mathfrak{H}}$-res.dim $K^{\prime \prime}=n-1$.

Proof. (1) If $n=1$, then there is an $\mathbb{E}$-triangle $X_{1} \rightarrow X_{0} \rightarrow M-\rightarrow$. By setting $K=X_{1}$ in the $\mathbb{E}$-triangles (11), we have $\mathfrak{X}$-res.dim $K=0$. If $n>1$, then $K \notin \mathfrak{X}$. Applying Corollary 1 (2) to the $\mathbb{E}$-triangle (11) yields that $\mathfrak{X}$-res.dim $K=n-1$. On the other hand, since $\mathfrak{H} \subseteq \mathfrak{X}$, we have $n-1=\mathfrak{X}$-res.dim $K \leq \mathfrak{H}$-res.dim $K \leq n-1$. Thus, $\mathfrak{H}$-res.dim $K=$ $n-1$.

Moreover, applying Corollary $1(1)$ to the $\mathbb{E}$-triangle (12) implies $\mathfrak{X}$-res.dim $W=$ $\mathfrak{X}$-res.dim $M=n$. Therefore, $n=\mathfrak{X}$-res.dim $W \leq \mathfrak{H}$-res.dim $W \leq n$. Hence $\mathfrak{H}$-res.dim $W=$ $\mathfrak{X}$-res.dim $W=n$.

The last assertion follows from the above argument and Proposition 4.

(2) Since $\mathfrak{X} \perp \mathfrak{H}$, we have $\mathfrak{X} \perp \widehat{\mathfrak{H}}$ by Lemma 3, and so the result immediately follows from (1) and Propostion 4.

(3) (a) $(\Leftarrow)$ Suppose $\mathfrak{X} \perp\left(\mathfrak{H}^{\perp} \cap \widehat{\mathfrak{H}}\right)$. Clearly, $\omega_{\mathfrak{H}}=\mathfrak{H}^{\perp} \cap \mathfrak{H} \subseteq \mathfrak{H}^{\perp} \cap \widehat{\mathfrak{H}} \subseteq \mathfrak{X}^{\perp}$, i.e., $\mathfrak{X} \perp \omega_{\mathfrak{H}}$.

$(\Rightarrow)$ Suppose $\mathfrak{X} \perp \omega_{\mathfrak{H}}$. Let $L \in \mathfrak{H}^{\perp} \cap \widehat{\mathfrak{H}}$. By Proposition 4 , there exists an $\mathbb{E}$-triangle

$$
K^{\prime} \longrightarrow H_{0} \longrightarrow L->
$$

in $\xi$ with $H_{0} \in \mathfrak{H}$ and $\omega_{\mathfrak{H}}$-res.dim $K^{\prime} \leq \mathfrak{H}$-res.dim $L-1<\infty$. Notice that $\mathfrak{H} \perp \omega_{\mathfrak{H}}$, so $\mathfrak{H} \perp \widehat{\omega_{\mathfrak{H}}}$ by Lemma 3 , and hence $K^{\prime} \in \mathfrak{H}^{\perp}$, thus $L \in \mathfrak{H}^{\perp}$ implies $H_{0} \in \mathfrak{H}^{\perp}$. Then $H_{0} \in \omega_{\mathfrak{H}}$, and so $L \in \widehat{\omega_{\mathfrak{H}}}$. Since $\mathfrak{X} \perp \omega_{\mathfrak{H}}$, we have $L \in \mathfrak{X}^{\perp}$ by Lemma 3. Thus, $\mathfrak{X} \perp\left(\mathfrak{H}^{\perp} \cap \widehat{\mathfrak{H}}\right)$.

(b) Suppose $\mathfrak{X}$-res.dim $M=n$, by (1) and Propostion 4, there exists an $\mathbb{E}$-triangle

$$
K \rightarrow X_{0} \rightarrow M-\rightarrow
$$

in $\tilde{\xi}$ with $\mathfrak{X}_{0} \in \mathfrak{X}$ and $\omega_{\mathfrak{X}}$-res.dim $K=n-1$. Notice that $\mathfrak{X} \perp \omega_{\mathfrak{X}}$, so $\mathfrak{X} \perp \widehat{\omega_{\mathfrak{X}}}$ by Lemma 3, thus $M \in \mathfrak{X}^{\perp}$ and $K \in \mathfrak{X}^{\perp}$, so $X_{0} \in \mathfrak{X}^{\perp}$ by Remark 4 , and hence $X_{0} \in \omega_{\mathfrak{X}}$. It follows that $\omega_{\mathfrak{X}}$-res.dim $M \leq n$. However, $n=\mathfrak{X}$-res.dim $M \leq \omega_{\mathfrak{X}}$-res.dim $M \leq n$, thus $\mathfrak{X}$-res.dim $M=\omega_{\mathfrak{X}}$-res.dim $M$.

(4) Suppose $\mathfrak{X}$-res.dim $M=n$, by (1), there exists an $\mathbb{E}$-triangle

$$
K \rightarrow X_{0} \rightarrow M->
$$

in $\xi$ with $\mathfrak{X}_{0} \in \mathfrak{X}$ and $\mathfrak{H}$-res.dim $K=n-1$. By (2), there is an $\mathbb{E}$-triangle

$$
K \longrightarrow K^{\prime \prime} \longrightarrow H->
$$

in $\xi$ with $H \in \mathfrak{H}, K^{\prime \prime} \in \mathfrak{H}^{\perp}$ and $\mathfrak{H}$-res.dim $K^{\prime \prime}=\mathfrak{H}$-res.dim $K$. Then $K^{\prime \prime} \in \mathfrak{H}^{\perp} \cap \widehat{\mathfrak{H}}$. By Lemma 1(2), we have the following commutative diagram

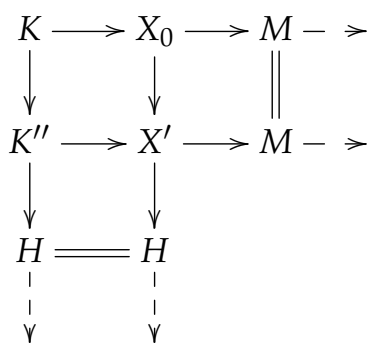

One can see that the $\mathbb{E}$-triangle

$$
K^{\prime \prime} \longrightarrow X^{\prime} \longrightarrow M->
$$


is in $\xi$ and $X^{\prime} \in \mathfrak{X}$. Notice that $\mathfrak{X} \perp \omega_{\mathfrak{H}}$, so $\mathfrak{X} \perp \mathfrak{H}^{\perp} \cap \widehat{\mathfrak{H}}$ by (3)(a). Then $\xi x t_{\tilde{\zeta}}^{1}\left(\widetilde{X}, K^{\prime \prime}\right)=0$ for any $\tilde{X} \in \mathfrak{X}$, and so $\operatorname{Hom}_{\mathcal{T}}\left(\widetilde{X}, X^{\prime}\right) \longrightarrow \operatorname{Hom}_{\mathcal{T}}(\widetilde{X}, M) \longrightarrow 0$ is exact. Thus, the $\xi$-deflation $X^{\prime} \longrightarrow M$ is a right $\mathfrak{X}$-approximation of $M$ and $\mathfrak{H}$-res.dim $K^{\prime \prime}=n-1$ in the $\mathbb{E}$-triangle (20). Notice that $K^{\prime \prime} \in \mathfrak{H}^{\perp}$, so we have $\omega_{\mathfrak{H}}$-res.dim $K^{\prime \prime}=\mathfrak{H}$-res.dim $K^{\prime \prime}=n-1$ by (3)(b).

Lemma 4. Let $\mathfrak{H}$ be a subcategory of $\mathscr{C}$ with $\mathfrak{H} \perp \mathfrak{H}$. Assume that $\mathfrak{H}$ is closed under cocones of $\xi$-deflations or closed under direct summands. Then $\mathfrak{H}=\widehat{\mathfrak{H}} \cap{ }^{\perp} \mathfrak{H}$.

Proof. Clearly, $\mathfrak{H} \subseteq \widehat{\mathfrak{H}} \cap{ }^{\perp} \mathfrak{H}$.

Conversely, let $M \in \widehat{\mathfrak{H}} \cap{ }^{\perp} \mathfrak{H}$. Then there is a $\xi$-exact complex

$$
H_{n} \stackrel{h_{n}}{\longrightarrow} H_{n-1} \stackrel{h_{n-1}}{\longrightarrow} \cdots \rightarrow H_{0} \stackrel{h_{0}}{\longrightarrow} M
$$

with each $H_{i} \in \mathfrak{H}$ for some nonnegative integer $n$. This means that there are $\mathbb{E}$-triangles $H_{n} \stackrel{h_{n}}{\longrightarrow} H_{n-1} \stackrel{g_{n-1}}{\longrightarrow} K_{n-1}->, K_{1} \stackrel{w_{0}}{\longrightarrow} H_{0} \stackrel{h_{0}}{\longrightarrow} M->$, and $K_{i+1} \stackrel{w_{i}}{\longrightarrow} H_{i} \stackrel{g_{i}}{\longrightarrow} K_{i}-\rightarrow$ in $\xi$ with $h_{i}=w_{i-1} g_{i}$ for any $0<i<n-1$. Then $M \in{ }^{\perp} \mathfrak{H}$ yields $K_{i} \in{ }^{\perp} \mathfrak{H}$ by Remark 4 , and so the $\mathbb{E}$-triangle

$$
H_{n} \stackrel{h_{n}}{\longrightarrow} H_{n-1} \stackrel{g_{n-1}}{\longrightarrow} K_{n-2}->
$$

is split. It follows that $H_{n-1} \cong H_{n} \oplus K_{n-2}$ and there exists an $\mathbb{E}$-triangle

$$
K_{n-2} \longrightarrow H_{n-1} \longrightarrow H_{n}-^{0}>
$$

in $\xi$. Since $\mathfrak{H}$ is closed under cocones of $\xi$-deflations or closed under direct summands by assumption, we have $K_{n-2} \in \mathfrak{H}$. Repeating this process, we can obtain $K_{i} \in \mathfrak{H}$, hence $M \in \mathfrak{H}$ and $\widehat{\mathfrak{H}} \cap{ }^{\perp} \mathfrak{H} \subseteq \mathfrak{H}$. Thus, $\widehat{\mathfrak{H}} \cap{ }^{\perp} \mathfrak{H}=\mathfrak{H}$.

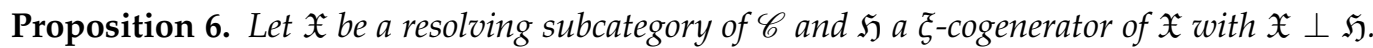
Assume that $\mathfrak{H}$ is closed under cocones of $\mathfrak{\xi}$-deflations or closed under direct summands. Then $\mathfrak{X}=\widehat{\mathfrak{X}} \cap \perp \widehat{\mathfrak{H}}=\widehat{\mathfrak{X}} \cap{ }^{\perp} \mathfrak{H}$.

Proof. Clearly, $\mathfrak{X} \subseteq \widehat{\mathfrak{X}} \cap{ }^{\perp} \mathfrak{H}$ and $\widehat{\mathfrak{X}} \cap \perp \widehat{\mathfrak{H}} \subseteq \widehat{\mathfrak{X}} \cap{ }^{\perp} \mathfrak{H}$.

Now, let $M \in \widehat{\mathfrak{X}} \cap{ }^{\perp} \mathfrak{H}$. Then by Lemma $3(2)$, we have $M \in \widehat{\mathfrak{X}} \cap{ }^{\perp} \widehat{\mathfrak{H}}$, and hence $\widehat{\mathfrak{X}} \cap{ }^{\perp} \mathfrak{H} \subseteq \widehat{\mathfrak{X}} \cap \perp \widehat{\mathfrak{H}}$.

On the other hand, by Proposition 4, there is an $\mathbb{E}$-triangle

$$
K \rightarrow X \rightarrow M->
$$

in $\xi$ with $X \in \mathfrak{X}$ and $\mathfrak{H}$-res.dim $K<\infty$. Notice that $M \in{ }^{\perp} \mathfrak{H}$ implies $K \in{ }^{\perp} \mathfrak{H}$ by Remark 4 , and hence $K \in \widehat{\mathfrak{H}} \cap{ }^{\perp} \mathfrak{H}=\mathfrak{H}$ by Lemma 4 . Notice that $\xi x t_{\tilde{\xi}}^{1}(M, K)=0$, so the $\mathbb{E}$-triangle (21) is split, hence $X \cong K \oplus M$. Consider the following $\mathbb{E}$-triangle

$$
M \rightarrow X \rightarrow K^{0}>
$$

in $\xi$. It follows that $M \in \mathfrak{X}$ from the assumption that $\mathfrak{X}$ is resolving. Thus, $\widehat{\mathfrak{X}} \cap{ }^{\perp} \mathfrak{H} \subseteq \mathfrak{X}$.

Our main result is the following

Theorem 2. Let $\mathfrak{X}$ be a resolving subcategory of $\mathscr{C}$ and $\mathfrak{H}$ a $\xi$-cogenerator of $\mathfrak{X}$ with $\mathfrak{X} \perp \mathfrak{H}$. Assume that $\mathfrak{H}$ is closed under cocones of $\mathfrak{\xi}$-deflations or closed under direct summands. For any $M \in \mathscr{C}$, if $M \in \widehat{\mathfrak{X}}$, then the following statements are equivalent:

(1) $\mathfrak{X}$-res.dim $M \leq m$.

(2) $\Omega^{n}(M) \in \mathfrak{X}$ for all $n \geq m$. 
(3) $\Omega_{\mathfrak{X}}^{n}(M) \in \mathfrak{X}$ for all $n \geq m$.

(4) $\xi x t_{\tilde{\xi}}^{n}(M, H)=0$ for all $n>m$ and all $H \in \mathfrak{H}$.

(5) $\xi x t_{\tilde{\zeta}}^{n}(M, L)=0$ for all $n>m$ and all $L \in \widehat{\mathfrak{H}}$.

(6) $M$ admits a right $\mathfrak{X}$-approximation $\varphi: X \rightarrow M$, where $\varphi$ is a $\xi$-deflation, such that there is an $\mathbb{E}$-triangle $K \longrightarrow X \stackrel{\varphi}{\longrightarrow} M->$ satisfying $\mathfrak{H}$-res.dim $K \leq m-1$.

(7) There are two $\mathbb{E}$-triangles

$$
W_{M} \rightarrow X_{M} \rightarrow M->
$$

and

$$
M \rightarrow W^{M} \longrightarrow X^{M}->
$$

in $\xi$ such that $X_{M}, \quad X^{M} \in \mathfrak{X}$ and $\mathfrak{H}$-res.dim $W_{M} \leq m-1$, $\mathfrak{H}$-res.dim $W^{M}=\mathfrak{X}$-res.dim $W^{M} \leq m$.

Proof. (1) $\Leftrightarrow(2) \Leftrightarrow$ (3) follow from Proposition 1 .

$(1) \Rightarrow(6)$ follows from Proposition 5(1), and (6) $\Rightarrow(1)$ is straightforward.

$(1) \Rightarrow(7)$ follows from Proposition 5(1), and (7) $\Rightarrow(1)$ is straightforward.

$(1) \Rightarrow(4)$ Suppose $\mathfrak{X}$-res.dim $M \leq m$. There is a $\xi$-exact complex

$$
X_{m} \stackrel{x_{m}}{\rightarrow} \cdots \rightarrow X_{0} \stackrel{x_{0}}{\rightarrow} M
$$

with all $X_{i}$ in $\mathfrak{X}$. This means that there are $\mathbb{E}$-triangles $X_{m} \stackrel{x_{m}}{\longrightarrow} X_{m-1} \stackrel{g_{m-1}}{\longrightarrow} K_{n-2}->$, $K_{1} \stackrel{w_{0}}{\longrightarrow} X_{0} \stackrel{x_{0}}{\longrightarrow} M->$, and $K_{i+1} \stackrel{w_{i}}{\longrightarrow} X_{i} \stackrel{g_{i}}{\longrightarrow} K_{i}->$ in $\xi$ with $x_{i}=w_{i-1} g_{i}$ for any $0<$ $i<m-1$. By assumption, we have $\xi x t_{\tilde{\xi}}^{k \geq 1}\left(X_{i}, H\right)=0$ for all $H \in \mathfrak{H}$. Thus, by Remark 4 , $\xi x t_{\tilde{\xi}}^{n}(M, H) \cong \xi x t_{\xi}^{n-m}\left(X_{m}, H\right)=0$ for $n>m$.

$(4) \Rightarrow$ (5) It follows from Lemma 3.

(5) $\Rightarrow(4)$ It is clear.

(4) $\Rightarrow(1)$ Since $M \in \widehat{\mathfrak{X}}$, by Proposition 5(1), there is an $\mathbb{E}$-triangle $K \longrightarrow X \rightarrow M->$ in $\xi$ with $\mathfrak{H}$-res.dim $K<\infty$ and $X \in \mathfrak{X}$. Then $\xi x t_{\xi}^{i}(K, H) \cong \xi x t_{\xi}^{i+1}(M, H)$ for $H \in \mathfrak{H}$ and $i \geq 1$ since $\xi x t_{\xi}^{i \geq 1}(X, H)=0$. Therefore, $\xi x t_{\xi}^{i \geq m}(K, H)=0$. Please note that $\mathfrak{H}$-res.dim $K<\infty$, so we have the following $\xi$-exact complex

$$
H_{n} \longrightarrow \cdots \rightarrow H_{0} \longrightarrow K
$$

with all $H_{i} \in \mathfrak{H}$. Then by Remark 4 ,

$$
\xi x t_{\xi}^{i}\left(\Omega_{\mathfrak{H}}^{m-1}(K), H\right) \cong \xi x t_{\mathcal{\xi}}^{i+m-1}(K, H)=0
$$

for $i \geq 1$ and all $H \in \mathfrak{H}$, which means $\Omega_{\mathfrak{H}}^{m-1}(K) \in{ }^{\perp} \mathfrak{H}$. Notice that $\mathfrak{H}$-res.dim $\Omega_{\mathfrak{H}}^{m-1}(K)<$ $\infty$, hence $\Omega_{\mathfrak{H}}^{m-1}(K) \in \widehat{\mathfrak{H}} \cap{ }^{\perp} \mathfrak{H}$. It follows that $\Omega_{\mathfrak{H}}^{m-1}(K) \in \mathfrak{H}$ from Lemma 4 , so $\mathfrak{H}$-res.dim $K \leq m-1$. Thus, $\mathfrak{X}$-res.dim $M \leq m$.

\section{Applications}

\subsection{Additive Quotient Categories with Respect to a Resolving Subcategory}

In this subsection, we will further study objects with a finite resolution dimension with respect to a resolving subcategory $\mathfrak{X}$. We construct adjoint pairs for two kinds of inclusion functors, which unifies some results of $[6,19]$.

Suppose that $\mathcal{D}$ and $\mathfrak{X}$ are two subcategories of $\mathscr{C}$. We use $[\mathcal{D}]$ to denote the ideal of $\mathfrak{X}$ consisting of morphisms which factor through some object in $\mathcal{D}$. Thus, one has a quotient category $\mathfrak{X} /[\mathcal{D}]$, which is also an additive category.

Throughout this subsection, we always assume that $\mathfrak{X}$ is a resolving subcategory of $\mathscr{C}$ and $\mathfrak{H}$ is a $\mathfrak{\xi}$-cogenerator of $\mathfrak{X}$ with $\mathfrak{X} \perp \mathfrak{H}$. 
Lemma 5. Let $f: X \rightarrow M$ be a morphism in $\mathscr{C}$ with $X \in \mathfrak{X}$ and $M \in \widehat{\mathfrak{X}}$. Then $f \in[\mathfrak{H}]$ if and only if $f \in[\widehat{\mathfrak{H}}]$.

Proof. The only if part is trivial.

For the if part, suppose that $f$ factors through an object $L \in \widehat{\mathfrak{H}}$. Then $f=g h$, where $h: X \rightarrow L$ and $g: L \rightarrow M$. Since $L \in \widehat{\mathfrak{H}}$, there is an $\mathbb{E}$-triangle

$$
L^{\prime} \longrightarrow H \rightarrow L-\rightarrow
$$

in $\bar{\xi}$ with $H \in \mathfrak{H}$ and $L^{\prime} \in \widehat{\mathfrak{H}}$. Notice that $\mathfrak{X} \perp \mathfrak{H}$, one has $\mathfrak{X} \perp \widehat{\mathfrak{H}}$ by Lemma 3 , and thus $\xi x t_{\tilde{\xi}}^{1}\left(X, L^{\prime}\right)=0$. Therefore, $h$ factors through $H$, it follows that $f$ factors through $H$.

Now let $M \in \widehat{\mathfrak{X}}$. By Proposition 4 , there is an $\mathbb{E}$-triangle

$$
W_{M} \stackrel{\alpha}{\longrightarrow} X_{M} \stackrel{p}{\longrightarrow} M->
$$

in $\xi$ with $X_{M} \in \mathfrak{X}$ and $W_{M} \in \widehat{\mathfrak{H}}$. Moreover, since we assume that $\mathfrak{X} \perp \mathfrak{H}$, the morphism $p$ is a right $\mathfrak{X}$-approximation of $M$ by Proposition 4 .

Lemma 6. Let $M, N \in \widehat{\mathfrak{X}}$ and $f: M \rightarrow N$ be a morphism in $\mathscr{C}$. Choose two $\mathbb{E}$-triangles

$$
W_{M} \stackrel{\alpha}{\rightarrow} X_{M} \stackrel{p}{\rightarrow} M-\rightarrow \text { and } W_{N} \stackrel{\beta}{\longrightarrow} X_{N} \stackrel{q}{\rightarrow} N->
$$

in $\xi$ with $X_{M}, X_{N} \in \mathfrak{X}$ and $W_{M}, W_{N} \in \widehat{\mathfrak{H}}$, then we have the following statements:

(1) There exists a morphism $g: X_{M} \rightarrow X_{N}$ such that $q g=f p$.

(2) If $g, g^{\prime}: X_{M} \rightarrow X_{N}$ are two morphisms such that $q g=f p$ and $q g^{\prime}=f p$, then $[g]=\left[g^{\prime}\right]$ in $\operatorname{Hom}_{\mathfrak{X} /[\mathfrak{H}]}\left(X_{M}, X_{N}\right)$.

Proof. (1) Since we assume that $\mathfrak{X} \perp \mathfrak{H}$, the morphism $p$ and $q$ are right $\mathfrak{X}$-approximation of $M$ and $N$ respectively by Proposition 4 . Then the existence of $g$ is obvious.

(2) Suppose $g, g^{\prime}: X_{M} \rightarrow X_{N}$ are two morphisms such that $q g=f p$ and $q g^{\prime}=f p$, then $q\left(g^{\prime}-g\right)=q g^{\prime}-q g=0$. By [12] of [Proposition 3.3], there exists a morphism $h: X_{M} \rightarrow W_{N}$ such that $g^{\prime}-g=\beta h$, i.e., there is a commutative diagram as follows:

$$
\begin{gathered}
X_{M} \\
W_{N} \stackrel{g^{\prime}-g}{\longrightarrow} X_{N} \stackrel{q}{\longrightarrow} N->.
\end{gathered}
$$

Notice that $W_{N} \in \widehat{\mathfrak{H}}$, so $g^{\prime}-g: X_{M} \rightarrow X_{N}$ factors through an object in $\mathfrak{H}$ by Lemma 5 . Thus, $[g]=\left[g^{\prime}\right]$ in $\operatorname{Hom}_{\mathfrak{X} /[\mathfrak{H}]}\left(X_{M}, X_{N}\right)$.

Now let

$$
W_{M} \stackrel{\alpha}{\rightarrow} X_{M} \stackrel{p}{\longrightarrow} M->\text { and } W_{M}^{\prime} \stackrel{\alpha^{\prime}}{\rightarrow} X_{M}^{\prime} \stackrel{p^{\prime}}{\longrightarrow} M->
$$

be two $\mathbb{E}$-triangles in $\xi$ with $X_{M}, X_{M}^{\prime} \in \mathfrak{X}$ and $W_{M}, W_{M}^{\prime} \in \widehat{\mathfrak{H}}$. By Lemma 6(1), there exist morphisms $g: X_{M} \rightarrow X_{M}^{\prime}$ and $h: X_{M}^{\prime} \rightarrow X_{M}$ such that $p^{\prime} g=\operatorname{Id}_{M} p=p$ and $p h=\operatorname{Id}_{M} p^{\prime}=p^{\prime}$. Thus, $p h g=p=p \operatorname{Id}_{M}$, and so $[h][g]=[h g]=\left[\operatorname{Id}_{M}\right]$ by Lemma 6(2). Similarly, $[g][h]=\left[\operatorname{Id}_{M}\right]$. Therefore, $X_{M} \cong X_{M}^{\prime}$ in $\mathfrak{X} /[\mathfrak{H}]$. Following this and Lemma 6(2), there exists a well-defined additive functor

$$
F: \widehat{\mathfrak{X}} \rightarrow \mathfrak{X} /[\mathfrak{H}]
$$


which maps an object $M \in \widehat{\hat{X}}$ to $X_{M}$ appeared in (22) and a morphism $f: M \rightarrow N \in$ $\operatorname{Hom}_{\widehat{\mathfrak{X}}}(M, N)$ to $[g] \in \operatorname{Hom}_{\mathfrak{X} /[\mathfrak{H}]}\left(X_{M}, X_{N}\right)$ as described in Lemma 6 .

It is obvious that $F(H)=0$ for any object $H \in \mathfrak{H}$. Hence, there exists an additive functor $\mu: \widehat{\mathfrak{X}} /[\mathfrak{H}] \rightarrow \mathfrak{X} /[\mathfrak{H}]$ making the following diagram

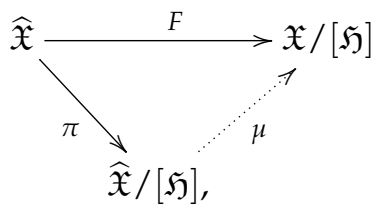

commute, where $\pi$ is the canonical quotient functor. Clearly, let $N \in \widehat{\mathcal{X}}$ and

$$
W_{N} \stackrel{\beta}{\rightarrow} X_{N} \stackrel{q}{\rightarrow} N->
$$

an $\mathbb{E}$-triangle in $\xi$ with $W_{N} \in \widehat{\mathfrak{H}}$ and $X_{N} \in \mathfrak{X}$, then $\mu(N)=\mu(\pi(N))=F(N)=X_{N}$.

We have the following result.

Theorem 3. The additive functor $\mu: \widehat{\mathfrak{X}} /[\mathfrak{H}] \rightarrow \mathfrak{X} /[\mathfrak{H}]$ defined above is right adjoint to the inclusion functor $\mathfrak{X} /[\mathfrak{H}] \rightarrow \widehat{\mathfrak{X}} /[\mathfrak{H}]$.

Proof. Let $X \in \mathfrak{X}$ and $N \in \widehat{\mathfrak{X}}$. Choose an $\mathbb{E}$-triangle

$$
W_{N} \stackrel{\beta}{\rightarrow} X_{N} \stackrel{q}{\rightarrow} N->
$$

in $\xi$ with $W_{N} \in \widehat{\mathfrak{H}}$ and $X_{N} \in \mathfrak{X}$. Notice that the additive map

$$
[q]_{\star}: \operatorname{Hom}_{\mathfrak{X} /[\mathfrak{H}]}(X, \mu(N)) \rightarrow \operatorname{Hom}_{\widehat{\mathfrak{X}} /[\mathfrak{H}]}(X, N)
$$

is natural in both $X$ and $N$ by Lemma 6 . We claim that $[q]_{\star}$ is an isomorphism.

Indeed, since $\mathfrak{X} \perp \mathfrak{H}$, by Lemma 3 , we have $\tilde{\xi} x t_{\tilde{\xi}}^{1}\left(X, W_{N}\right)=0$, and hence $\operatorname{Hom}_{\mathscr{C}}\left(X, X_{N}\right) \rightarrow \operatorname{Hom}_{\mathscr{C}}(X, N)$ is an epimorphism, so $[q]_{\star}$ is still an epimorphism.

Now, assume that $g: X \rightarrow X_{N}$ is a morphism such that $[q g]=[q][g]=[q]_{\star}[g]=$ $[0] \in \operatorname{Hom}_{\widehat{X} /[\mathfrak{H}]}(X, N)$. Then there exist an object $H \in \mathfrak{H}$ and morphisms $s: X \rightarrow H$ and $t: H \rightarrow N$ such that $q g=t$ s. Consider the following diagram below

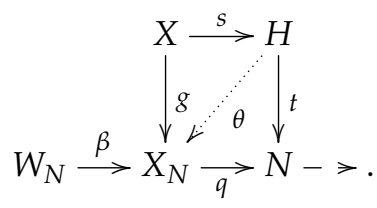

Since $\mathfrak{X} \perp \mathfrak{H}$ by assumption, one has $\mathfrak{X} \perp \widehat{\mathfrak{H}}$ by Lemma 3 . Moreover, since $\mathfrak{H} \subseteq \mathfrak{X}, \mathfrak{H} \perp \widehat{\mathfrak{H}}$, and hence $\xi x t_{\tilde{\xi}}^{1}\left(H, W_{N}\right)=0$. Thus, there exists a morphism $\theta: H \rightarrow X_{N}$ such that $t=q \theta$. Since $q(g-\theta s)=q g-q \theta s=t s-t s=0$, so $g-\theta s$ factors through $W_{N}$. By Lemma 5 , $g-\theta s$ factors through an object in $\mathfrak{H}$. It follows that $[g-\theta s]=0 \in \operatorname{Hom}_{\mathfrak{X} /[\mathfrak{H}]}(X, N)$. Since $\theta s=0 \in \operatorname{Hom}_{\mathfrak{X} /[\mathfrak{H}]}(X, N)$, we have $0=[g] \in \operatorname{Hom}_{\mathfrak{X} /[\mathfrak{H}]}(X, N)$. Therefore, $[q]_{\star}$ is a monomorphism, and thus $[q]_{\star}$ is an isomorphism.

Corollary 2. Assume that $\mathfrak{H}$ is closed under direct summands. For any $N \in \widehat{\mathfrak{X}}$, the following statements are equivalent:

(1) $N \in \widehat{\mathfrak{H}}$. 
(2) There is an $\mathbb{E}$-triangle

$$
W_{N} \rightarrow X_{N} \stackrel{q}{\rightarrow} N->
$$

in $\xi$ with $W_{N} \in \widehat{\mathfrak{H}}$ and $X_{N} \in \mathfrak{X}$ such that $[q]=[0] \in \operatorname{Hom}_{\widehat{\mathfrak{X}} /[\mathfrak{H}]}(X, N)$.

Proof. The assertion (1) $\Rightarrow(2)$ follows from Lemma 5. It suffices to show (2) $\Rightarrow(1)$. Notice that the adjunction isomorphism established in Theorem 3 implies that the additive map

$$
[q]_{\star}: \operatorname{Hom}_{\mathfrak{X} /[\mathfrak{H}]}\left(X_{N}, X_{N}\right) \rightarrow \operatorname{Hom}_{\widehat{X} /[\mathfrak{H}]}\left(X_{N}, N\right)
$$

is an isomorphism. Since $[q]_{\star}\left[\operatorname{id}_{X_{N}}\right]=\left[q \operatorname{id}_{X_{N}}\right]=[q]=[0] \in \operatorname{Hom}_{\widehat{\mathcal{X}} /[\mathfrak{H}]}\left(X_{N}, N\right)=0$, so $\left[\operatorname{id}_{X_{N}}\right]=[0] \in \operatorname{Hom}_{\widehat{X} /[\mathfrak{H}]}\left(X_{N}, X_{N}\right)$, and thus $\operatorname{id}_{X_{N}}$ factors through an object $H \in \mathfrak{H}$. It follows that $X_{N}$ is a direct summand of $H$. Since $\mathfrak{H}$ is closed under direct summands, we have $X_{N} \in \mathfrak{H}$. Thus, $N \in \widehat{\mathfrak{H}}$.

Next we compare additive quotients $\widehat{\mathfrak{H}} /[\mathfrak{X}]$ and $\widehat{\mathfrak{X}} /[\mathfrak{X}]$. Let $M \in \widehat{\mathfrak{X}}$. By Proposition 4 , there is an $\mathbb{E}$-triangle

$$
M \stackrel{s}{\rightarrow} W^{M} \stackrel{l}{\longrightarrow} X^{M}->
$$

in $\tilde{\xi}$ with $X^{M} \in \mathfrak{X}$ and $W^{M} \in \widehat{\mathfrak{H}}$. Moreover, since we assume that $\mathfrak{X} \perp \mathfrak{H}$, the morphism $s$ is a left $\widehat{\mathfrak{H}}$-approximation of $M$ by Proposition 4 .

Lemma 7. Let $M, N \in \widehat{\mathfrak{X}}$ and $f: M \rightarrow N$ be a morphism in $\mathscr{C}$. Choose two $\mathbb{E}$-triangles

$$
M \stackrel{s}{\rightarrow} W^{M} \stackrel{l}{\rightarrow} X^{M}->\text { and } N \stackrel{t}{\rightarrow} W^{N} \stackrel{r}{\rightarrow} X^{N}->
$$

in $\xi$ with $X^{M}, X^{N} \in \mathfrak{X}$ and $W^{M}, W^{N} \in \widehat{\mathfrak{H}}$. Then we have the following statements:

(1) There exists a morphism $g: W^{M} \rightarrow W^{N}$ such that $g s=t f$.

(2) If $g, g^{\prime}: W^{M} \rightarrow W^{N}$ are two morphisms such that $g s=t f$ and $g^{\prime} s=t f$, then $[g]=\left[g^{\prime}\right]$ in $\operatorname{Hom}_{\widehat{\mathfrak{H}} /[\mathfrak{X}]}\left(X_{M}, X_{N}\right)$.

Proof. (1) Since $\mathfrak{X} \perp \mathfrak{H}$ by assumption, we have $\xi x t_{\tilde{\xi}}^{1}\left(X^{M}, W^{N}\right)=0$ by Lemma 3. Therefore, there exists a morphism $g: W^{M} \rightarrow W^{N}$ such that $g_{s}=t f$.

(2) Suppose $g, g^{\prime}: W^{M} \rightarrow W^{N}$ are two morphisms such that $g s=t f$ and $g^{\prime} s=t f$, then $\left(g^{\prime}-g\right) s=g^{\prime} s-g s=0$, and so there exists a morphism $h^{\prime}: X^{M} \rightarrow W^{N}$ such that $g^{\prime}-g=h^{\prime} l$, i.e., there is a commutative diagram as follows:

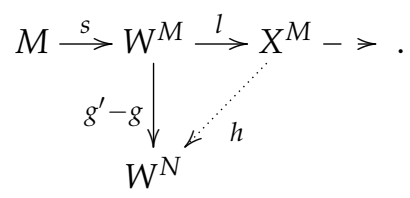

Notice that $X^{M} \in \mathfrak{X}$, so $g^{\prime}-g: W^{M} \rightarrow W^{N}$ factors through an object in $\mathfrak{X}$. Thus, $[g]=\left[g^{\prime}\right] \operatorname{in}_{\widehat{H}} \operatorname{Hom}_{\widehat{\mathfrak{H}} /[\mathfrak{X}]}\left(W^{M}, W^{N}\right)$.

As a similar argument to the functor $F$, by Lemma 7 , there exists a well-defined additive functor

$$
G: \widehat{\mathfrak{X}} \rightarrow \widehat{\mathfrak{H}} /[\mathfrak{X}]
$$

which maps an object $M \in \widehat{\mathfrak{X}}$ to $W^{M}$ appeared in (23) and a morphism $f: M \rightarrow N \in$ $\operatorname{Hom}_{\widehat{\mathfrak{X}}}(M, N)$ to $[g] \in \operatorname{Hom}_{\widehat{\mathfrak{H}} /[\mathfrak{X}]}\left(W^{M}, W^{N}\right)$ as described in Lemma 7 . 
Obviously, $G(X)=0$ for any object $X \in \mathfrak{X}$. Hence, there exists an additive functor $\eta: \widehat{\mathfrak{X}} /[\mathfrak{X}] \rightarrow \widehat{\mathfrak{H}} /[\mathfrak{X}]$ making the following diagram commute

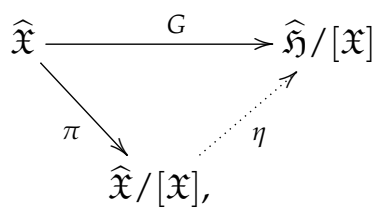

where $\eta$ is the canonical quotient functor.

We have the following result.

Theorem 4. The additive functor $\eta: \widehat{\mathfrak{X}} /[\mathfrak{X}] \rightarrow \widehat{\mathfrak{H}} /[\mathfrak{X}]$ defined above is left adjoint to the inclusion functor $\widehat{\mathfrak{H}} /[\mathfrak{X}] \rightarrow \widehat{\mathfrak{X}} /[\mathfrak{X}]$.

Proof. Let $K$ be an object in $\widehat{\mathfrak{H}}$ and $M$ an object in $\widehat{\mathfrak{X}}$. By Proposition 4 , there is an $\mathbb{E}$-triangle

$$
M \stackrel{s}{\rightarrow} W^{M} \stackrel{l}{\longrightarrow} X^{M}->
$$

in $\xi$ with $W^{M} \in \widehat{\mathfrak{H}}$ and $X^{M} \in \mathfrak{X}$. Notice that the additive map

$$
[s]^{\star}: \operatorname{Hom}_{\widehat{\mathfrak{H}} /[\mathfrak{X}]}(\eta(M), K) \longrightarrow \operatorname{Hom}_{\widehat{\mathfrak{X}} / \mathfrak{X}}(M, K)
$$

is natural in both $M$ and $K$ by Lemma 7 . We claim that $[s]^{\star}$ is an isomorphism.

Indeed, since $\mathfrak{X} \perp \mathfrak{H}$, by Lemma 3 , we have $\xi x t_{\tilde{\xi}}^{1}\left(X^{M}, K\right)=0$, and hence $\operatorname{Hom}_{\mathscr{C}}\left(W^{M}, K\right) \rightarrow \operatorname{Hom}_{\mathscr{C}}(M, K)$ is an epimorphism, so $[s]^{\star}$ is still an epimorphism.

Now, assume that $g: W^{M} \rightarrow K$ is a morphism such that $[g s]=[g][s]=[s]^{\star}[g]=$ $[0] \in \operatorname{Hom}_{\widehat{\mathfrak{X}} /[\mathfrak{X}]}(M, K)$. Then there exist an object $X \in \mathfrak{X}$ and morphisms $v: M \rightarrow X$ and $k: X \rightarrow K$ such that $g s=k v$. Since $\mathfrak{H}$ is a $\xi$-cogenerator of $\mathfrak{X}$, there exists an $\mathbb{E}$-triangle

$$
X \rightarrow H \rightarrow X^{\prime}->
$$

in $\xi$ with $H \in \mathfrak{H}$ and $X^{\prime} \in \mathfrak{X}$.

Notice that $\xi x t_{\xi}^{1}\left(X^{M}, H\right)=0$ and $\xi x t_{\xi}^{1}\left(X^{\prime}, K\right)=0$, by Remark 4 we get the following commutative diagram

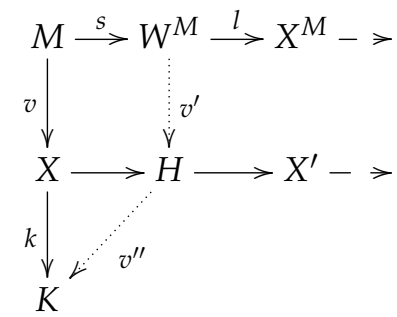

It follows that $\left[v^{\prime \prime} v^{\prime}\right]=[0] \in \operatorname{Hom}_{\widehat{\mathfrak{H}} / \mathfrak{X}}\left(W^{M}, K\right)$ as $H \in \mathfrak{X}$. Since $v^{\prime \prime} v^{\prime} s=k v=g s \in$ $\operatorname{Hom}_{\widehat{\mathfrak{H}} /[\mathfrak{X}]}(M, K)$, by Lemma $7(2)$, we have $[g]=\left[v^{\prime \prime} v^{\prime}\right] \in \operatorname{Hom}_{\widehat{\mathfrak{H}} /[\mathfrak{X}]}\left(W^{M}, K\right)$, and hence $[g]=0$. Therefore, $[s]^{\star}$ is a monomorphism, and thus $[s]^{\star}$ is an isomorphism.

Corollary 3. Assume that $\mathfrak{X}$ is closed under direct summands. For any $N \in \widehat{\mathfrak{X}}$, the following statements are equivalent:

(1) $N \in \mathfrak{X}$

(2) There is an $\mathbb{E}$-triangle

$$
N \stackrel{s}{\rightarrow} W^{N} \longrightarrow X^{N}->
$$

in $\xi$ with $W^{N} \in \widehat{\mathfrak{H}}$ and $X^{N} \in \mathfrak{X}$ such that $[s]=[0] \in \operatorname{Hom}_{\widehat{\mathfrak{X}} /[\mathfrak{X}]}\left(N, W^{N}\right)$. 
Proof. The assertion $(1) \Rightarrow(2)$ is obvious. It suffices to show $(2) \Rightarrow(1)$. Notice that the adjunction isomorphism established in Theorem 4 implies that the additive map

$$
[s]^{\star}: \operatorname{Hom}_{\widehat{\mathfrak{H}} /[\mathfrak{X}]}\left(W^{N}, W^{N}\right) \rightarrow \operatorname{Hom}_{\widehat{\mathfrak{X}} / \mathfrak{X}}\left(N, W^{N}\right)
$$

is isomorphic. Since $[s]^{\star}\left[\mathrm{id}_{W^{N}}\right]=\left[\operatorname{id}_{W^{N}} s\right]=[s]=[0] \in \operatorname{Hom}_{\widehat{X} /[\mathfrak{X}]}\left(N, W^{N}\right)=0$, so $\left[\operatorname{id}_{W^{N}}\right]=[0] \in \operatorname{Hom}_{\widehat{\mathfrak{H}} /[\mathfrak{X}]}\left(W^{N}, W^{N}\right)$, and thus $\operatorname{id}_{W^{N}}$ factors through an object $X^{\prime} \in \mathfrak{X}$. It follows that $W^{N}$ is a direct summand of $X^{\prime}$. Since $\mathfrak{X}$ is closed under direct summands, we have $W^{N} \in \mathfrak{X}$. Thus, $N \in \mathfrak{X}$.

\subsection{Construct a New Resolving Subcategory}

In this subsection, we will construct a new resolving subcategory from a given resolving subcategory, which generalizes the notion of $\xi$-Gorenstein projective objects given by $\mathrm{Hu}$, Zhang and Zhou [13]. By applying the results of this section to the subcategory of $\xi$ - $\mathcal{G}$ projective objects, we reformulate some known results in $[8,13]$.

Definition 19. Let $\mathfrak{X}$ be a subcategory of $\mathscr{C}$ and $M \in \mathscr{C}$. A complete $\mathcal{P}(\mathfrak{\xi}) \mathfrak{X}$-resolution of $M$ is $a \operatorname{Hom}_{\mathscr{C}}(-, \mathfrak{X})$-exact $\xi$-exact complex

$$
\cdots \rightarrow P_{1} \rightarrow P_{0} \rightarrow X^{0} \rightarrow X^{1} \rightarrow \cdots
$$

in $\mathscr{C}$ with all $P_{i} \in \mathcal{P}(\mathfrak{\xi}), X^{i} \in \mathfrak{X} \cap{ }^{\perp} \mathfrak{X}$ such that both

$$
K_{1} \longrightarrow P_{0} \longrightarrow M->\text { and } M \rightarrow X^{0} \rightarrow K^{1}->
$$

are corresponding $\mathbb{E}$-triangles in $\xi$. The $\mathcal{G} \mathcal{P}_{\mathfrak{X}}(\xi)$-Gorenstein category is defined as

$$
\mathcal{G} \mathcal{P}_{\mathfrak{X}}(\xi)=\{M \in \mathscr{C} \mid M \text { admits a complete } \mathcal{P}(\mathfrak{\xi}) \mathfrak{X} \text {-resolution }\} .
$$

Throughout this subsection, we always assume that $\mathfrak{X}$ is a resolving subcategory of $\mathscr{C}$.

Remark 7. (1) Since $\mathfrak{X}$ is a resolving subcategory of $\mathscr{C}$, we have $\mathcal{P}(\xi) \subseteq \mathfrak{X}$, so $\mathcal{P}(\xi) \subseteq \mathfrak{X} \cap{ }^{\perp} \mathfrak{X}$. Then we have $K_{1} \in \mathcal{G} \mathcal{P}_{\mathfrak{X}}(\xi)$.

(2) If $M \in \mathcal{G} \mathcal{P}_{\mathfrak{X}}(\xi)$, then $\xi x t_{\tilde{\xi}}^{0}(M, X) \cong \operatorname{Hom}_{\mathscr{C}}(M, X)$ and $\xi x t_{\tilde{\xi}}^{\geq 1}(M, X)=0$ for any $X \in \mathfrak{X}$. Indeed, let $M \in \mathcal{G} \mathcal{P}_{\mathfrak{X}}(\xi)$, then there is an $\mathbb{E}$-triangle

$$
K_{1} \longrightarrow P_{0} \longrightarrow M->
$$

in $\xi$ with $P_{0} \in \mathcal{P}(\xi)$ and $K_{1} \in \mathcal{G} \mathcal{P}_{\mathfrak{X}}(\xi)$, which is $\operatorname{Hom}_{\mathscr{C}}(-, \mathfrak{X})$-exact. If $X \in \mathfrak{X}$, then we have the following commutative diagram

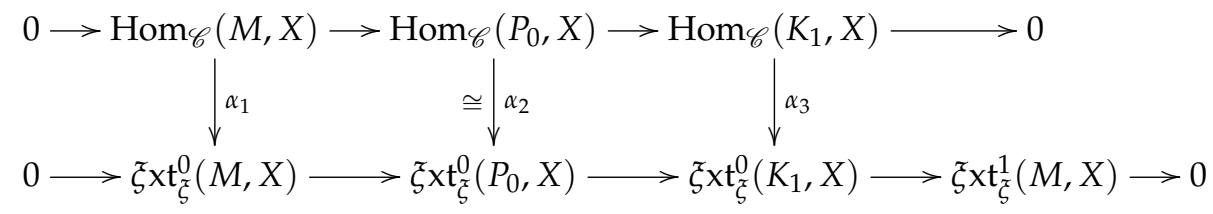

Using the proof of [8] of [Lemma 3.6], we have $\xi x t_{\tilde{\xi}}^{0}(M, X) \cong \operatorname{Hom}_{\mathscr{C}}(M, X)$. Moreover, there is a $\operatorname{Hom}_{\mathscr{C}}(-, \mathfrak{X})$-exact $\xi$-exact complex

$$
\cdots \rightarrow P_{1} \longrightarrow P_{0} \rightarrow M
$$

which is a $\xi$-projective resolution of $M$, thus $\xi x t_{\tilde{\xi}}^{\geq 1}(M, X)=0$ for any $X \in \mathfrak{X}$. 
Evidently, since $\mathscr{C}$ has enough $\xi$-projectives, $M \in \mathcal{G} \mathcal{P}_{\mathfrak{X}}(\xi)$ if and only if $\xi x t_{\tilde{\xi}}^{0}(M, X) \cong$ $\operatorname{Hom}_{\mathscr{C}}(M, X)$ and $\xi x t_{\tilde{\zeta}}^{>1}(M, X)=0$ for any $X \in \mathfrak{X}$, and $M$ admits $a \operatorname{Hom}_{\mathscr{C}}(-, \mathfrak{X})$-exact $\xi$-exact complex

$$
M \rightarrow X^{0} \rightarrow X^{1} \rightarrow \cdots
$$

with $X^{i} \in \mathfrak{X} \cap{ }^{\perp} \mathfrak{X}$.

(3) If $\mathfrak{X}=\mathcal{P}(\xi)$, then clearly $\mathfrak{X} \cap{ }^{\perp} \mathfrak{X}=\mathcal{P}(\xi)$, and thus $\mathcal{G} \mathcal{P} \mathfrak{X}(\xi)$ coincides with $\mathcal{G} \mathcal{P}(\xi)$ defined in [13].

Lemma 8. Assume $\mathfrak{X} \cap{ }^{\perp} \mathfrak{X} \subseteq \mathcal{G} \mathcal{P}_{\mathfrak{X}}(\mathfrak{\xi})$. Then $\mathfrak{X} \cap{ }^{\perp} \mathfrak{X}$ is a $\xi$-cogenerator of $\mathcal{G} \mathcal{P}_{\mathfrak{X}}(\xi)$ and is closed under cocones of $\xi$-deflations.

Proof. Let $M \in \mathcal{G} \mathcal{P}_{\mathfrak{X}}(\xi)$. There is a $\operatorname{Hom}_{\mathscr{C}}(-, \mathfrak{X})$-exact $\mathbb{E}$-triangle

$$
M \rightarrow X^{0} \rightarrow K^{1}->
$$

in $\xi$ with $X^{0} \in \mathfrak{X} \cap{ }^{\perp} \mathfrak{X} \subseteq \mathcal{G} \mathcal{P}_{\mathfrak{X}}(\xi)$. For any $X \in \mathfrak{X}$, applying the functor $\operatorname{Hom}_{\mathscr{C}}(-, X)$ to the $\mathbb{E}$-triangle (24) yields the following commutative diagram

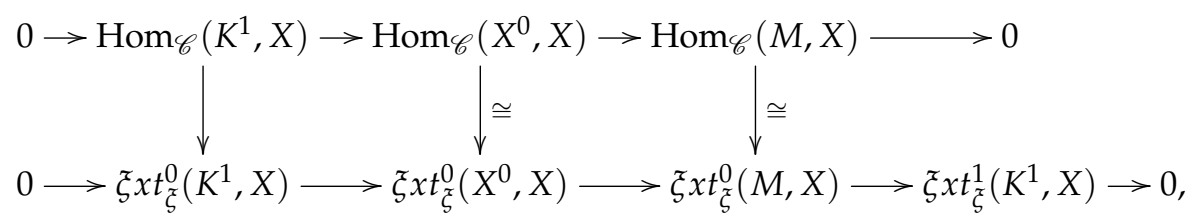

where the two isomorphisms follows from the assumption that $X^{0}, M \in \mathcal{G} \mathcal{P}_{\mathfrak{X}}(\mathfrak{\zeta})$ and Remark 7(2). It follows that $\xi x t_{\tilde{\xi}}^{1}\left(K^{1}, X\right)=0$ and $\xi x t_{\tilde{\xi}}^{0}\left(K^{1}, X\right) \cong \operatorname{Hom}_{\mathscr{C}}\left(K^{1}, X\right)$, so $K^{1} \in$ $\mathcal{G} \mathcal{P}_{\mathfrak{X}}(\xi)$ by Remark 7(2), then $\mathfrak{X} \cap{ }^{\perp} \mathfrak{X}$ is a $\xi$-cogenerator of $\mathcal{G} \mathcal{P}_{\mathfrak{X}}(\xi)$.

It is obvious that $\mathfrak{X} \cap \perp \mathfrak{X}$ is closed under cocones of $\xi$-deflations.

We have the following result.

Theorem 5. Assume $\mathfrak{X} \cap{ }^{\perp} \mathfrak{X} \subseteq \mathcal{G} \mathcal{P}_{\mathfrak{X}}(\xi)$. The subcategory $\mathcal{G} \mathcal{P}_{\mathfrak{X}}(\xi)$ is a resolving subcategory of $\mathscr{C}$.

Proof. The step 1: Let $P$ be a $\xi$-projective object. Consider the following $\xi$-exact complex

$$
\cdots \rightarrow 0 \stackrel{0}{\rightarrow} P \stackrel{\operatorname{id}_{P}}{\longrightarrow} P \stackrel{0}{\longrightarrow} 0 \rightarrow \cdots
$$

in $\mathscr{C}$. Clearly, it is $\operatorname{Hom}_{\mathscr{C}}(-, \mathfrak{X})$-exact. In particular,

$$
0 \stackrel{0}{\rightarrow} P \stackrel{\mathrm{id}_{P}}{\longrightarrow} P{ }_{-}^{0} \rightarrow \text { and } P \stackrel{\mathrm{id}_{P}}{\longrightarrow} P \stackrel{0}{\longrightarrow} 0{ }_{-}^{0}>
$$

are corresponding $\mathbb{E}$-triangles in $\xi$. Since $P \in \mathfrak{X} \cap{ }^{\perp} \mathfrak{X}$ by Remark $7(1)$, we have $\mathcal{P}(\xi) \subseteq \mathcal{G} \mathcal{P}_{\mathfrak{X}}(\xi)$.

The step 2: Given any $\mathbb{E}$-triangle

$$
M_{1} \stackrel{u}{\longrightarrow} M_{2} \stackrel{v}{\longrightarrow} M_{3}->\text {. }
$$

in $\xi$ with $M_{1}, M_{3} \in \mathcal{G} \mathcal{P}_{\mathfrak{X}}(\xi)$. Then

(i) $\xi x t_{\tilde{\xi}}^{0}\left(M_{1}, X\right) \cong \operatorname{Hom}_{\mathscr{C}}\left(M_{1}, X\right)$ and $\xi x t_{\tilde{\xi}}^{\geq 1}\left(M_{1}, X\right)=0$ for any $X \in \mathfrak{X}$, and $M_{1}$ admits

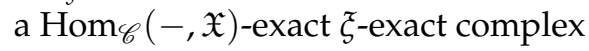

$$
M_{1} \longrightarrow X_{1}^{0} \longrightarrow X_{1}^{1} \rightarrow \cdots
$$

with $X_{1}^{i} \in \mathfrak{X} \cap{ }^{\perp} \mathfrak{X}$. 
(ii) $\xi x t_{\tilde{\xi}}^{0}\left(M_{3}, X\right) \cong \operatorname{Hom}_{\mathscr{C}}\left(M_{3}, X\right)$ and $\xi x t_{\tilde{\xi}}^{\geq 1}\left(M_{3}, X\right)=0$ for any $X \in \mathfrak{X}$, and $M_{3}$ admits a $\operatorname{Hom}_{\mathscr{C}}(-, \mathfrak{X})$-exact $\xi$-exact complex

$$
M_{3} \rightarrow X_{3}^{0} \rightarrow X_{3}^{1} \longrightarrow \cdots
$$

with $X_{3}^{i} \in \mathfrak{X} \cap{ }^{\perp} \mathfrak{X}$.

Applying $\operatorname{Hom}_{\mathscr{C}}(-, X)$ for any $X \in \mathfrak{X}$, we have a commutative diagram as follows

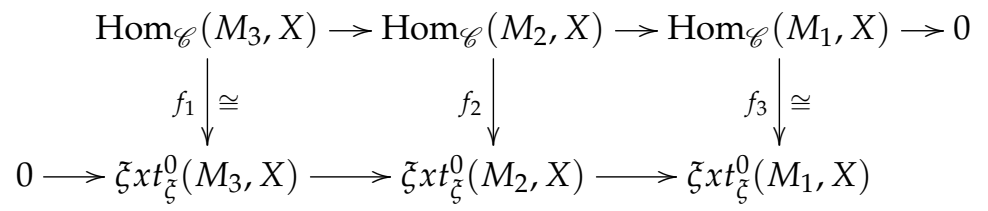

with exact rows. By (i) and (ii), $f_{1}$ and $f_{3}$ are isomorphic, so $f_{2}$ is isomorphic, i.e., $\xi x t_{\tilde{\xi}}^{0}\left(M_{2}, X\right) \cong \operatorname{Hom}_{\mathscr{C}}\left(M_{2}, X\right)$. Moreover, by Remark 4 , we have $\xi x t_{\bar{\xi}}^{\geq 1}\left(M_{2}, X\right)=0$ for any $X \in \mathfrak{X}$. Finally, the $\mathbb{E}$-triangle (26) is $\operatorname{Hom}_{\mathscr{C}}\left(-, \mathfrak{X} \cap{ }^{\perp} \mathfrak{X}\right)$-exact, so using a similar argument to that of the Horseshoe Lemma (e.g., [13] of [Theorem 4.16]), we can construct a $\operatorname{Hom}_{\mathscr{C}}(-, \mathfrak{X})$-exact $\mathfrak{\xi}$-exact complex

$$
M_{2} \rightarrow X_{2}^{0} \rightarrow X_{2}^{1} \rightarrow \cdots
$$

with $X_{2}^{i} \in \mathfrak{X} \cap{ }^{\perp} \mathfrak{X}$. Thus, $M_{2} \in \mathcal{G} \mathcal{P}_{\mathfrak{X}}(\mathfrak{\xi})$.

The step 3: Given any $\mathbb{E}$-triangle

$$
M_{1} \stackrel{u}{\longrightarrow} M_{2} \stackrel{v}{\longrightarrow} M_{3}->
$$

in $\xi$ with $M_{2}, M_{3} \in \mathcal{G} \mathcal{P}_{\mathfrak{X}}(\xi)$. As a similar argument to that of the step 2, we can prove that $\xi x t_{\tilde{\xi}}^{0}\left(M_{1}, X\right) \cong \operatorname{Hom}_{\mathscr{C}}\left(M_{1}, X\right)$ and $\xi x t_{\bar{\xi}}^{\geq 1}\left(M_{1}, X\right)=0$ for any $X \in \mathfrak{X}$. Moreover, since $\xi x t_{\tilde{\zeta}}^{0}\left(M_{2}, X\right) \cong \operatorname{Hom}_{\mathscr{C}}\left(M_{2}, X\right)$ and $\xi x t_{\tilde{\xi}}^{0}\left(M_{3}, X\right) \cong \operatorname{Hom}_{\mathscr{C}}\left(M_{3}, X\right)$ for any $X \in \mathfrak{X}$, it follows from Remark 4 that the $\mathbb{E}$-triangle (26) is $\operatorname{Hom}_{\mathscr{C}}(-, \mathfrak{X})$-exact. By Lemma 8 , there is an $\mathbb{E}$-triangle $M_{2} \stackrel{u}{\longrightarrow} X_{1}^{0} \stackrel{v}{\longrightarrow} K_{2}^{1}->$ in $\xi$ with $X_{1}^{0} \in \mathfrak{X} \cap{ }^{\perp} \mathfrak{X}$ and $K_{2}^{1} \in \mathcal{G} \mathcal{P}_{\mathfrak{X}}(\xi)$. By (ET4), we have the following commutative diagram

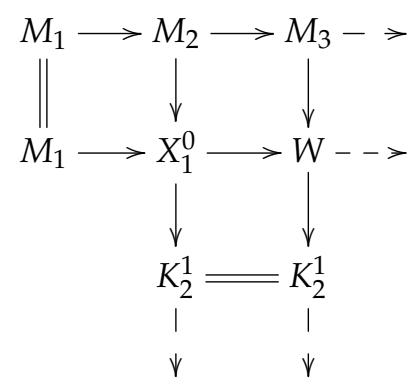

Since $M_{3}, K_{2}^{1} \in \mathcal{G} \mathcal{P}_{\mathfrak{X}}(\xi)$, we have $W \in \mathcal{G} \mathcal{P}_{\mathfrak{X}}(\xi)$ by the step 2, and the $\mathbb{E}$-triangle $M_{1} \longrightarrow X_{1}^{0} \longrightarrow W->$ in $\xi$ is $\operatorname{Hom}_{\mathscr{C}}(-, \mathfrak{X})$-exact. Thus, we can get a $\operatorname{Hom}_{\mathscr{C}}(-, \mathfrak{X})$-exact $\xi$-exact complex

$$
M_{1} \rightarrow X_{1}^{0} \rightarrow X_{1}^{1} \rightarrow \cdots
$$

with $X_{1}^{i} \in \mathfrak{X} \cap{ }^{\perp} \mathfrak{X}$. Thus, $M_{1} \in \mathcal{G P} \mathfrak{X}(\xi)$.

Therefore, $\mathcal{G} \mathcal{P}_{\mathfrak{X}}(\xi)$ is a resolving subcategory of $\mathscr{C}$.

As an application of Theorem 2, we have 
Proposition 7. Assume $\mathfrak{X} \cap{ }^{\perp} \mathfrak{X} \subseteq \mathcal{G} \mathcal{P}_{\mathfrak{X}}(\mathfrak{\xi})$. If $\left.M \in \widehat{\mathcal{G}} \widehat{\mathcal{P}_{\mathfrak{X}}(\xi)}\right)$, then the following statements are equivalent:

(1) $\mathcal{G} \mathcal{P}_{\mathfrak{X}}(\xi)$-res.dim $M \leq m$.

(2) $\Omega^{n}(M) \in \mathcal{G} \mathcal{P}_{\mathfrak{X}}(\xi)$ for all $n \geq m$.

(3) $\Omega_{\mathcal{G} \mathcal{P}_{\mathfrak{X}}(\xi)}^{n}(M) \in \mathcal{G} \mathcal{P}_{\mathfrak{X}}(\xi)$ for all $n \geq m$.

(4) $\xi x t_{\tilde{\xi}}^{n}(M, H)=0$ for all $n>m$ and all $H \in \mathfrak{X} \cap{ }^{\perp} \mathfrak{X}$.

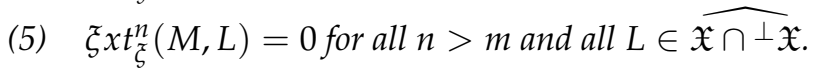

(6) $\quad M$ admits a right $\mathcal{G} \mathcal{P}_{\mathfrak{X}}(\xi)$-approximation $\varphi: X \rightarrow M$, where $\varphi$ is a $\xi$-deflation, such that there is an $\mathbb{E}$-triangle $K \longrightarrow X \stackrel{\varphi}{\rightarrow} M->$ satisfying $\mathfrak{H}$-res.dim $K \leq m-1$.

(7) There are two $\mathbb{E}$-triangles

$$
W_{M} \rightarrow X_{M} \rightarrow M->
$$

and

$$
M \rightarrow W^{M} \rightarrow X^{M}->
$$

in $\xi$ such that $X_{M}, X^{M} \in \mathcal{G} \mathcal{P}_{\mathfrak{X}}(\xi)$ and $\mathfrak{X} \cap{ }^{\perp} \mathfrak{X}$-res.dim $W_{M} \leq m-1$, $\mathfrak{X} \cap{ }^{\perp} \mathfrak{X}$-res.dim $W^{M}=\mathcal{G} \mathcal{P}_{\mathfrak{X}}(\mathfrak{\xi})$-res.dim $W^{M} \leq m$.

Putting $\mathfrak{X}=\mathcal{P}(\xi)$, we have the following corollary immediately, which reformulates and generalizes [13] of [Proposition 5.9] and [8] of [Theorem 3.9].

Corollary 4. Let $M \in \mathscr{C}$. If $M \in \widehat{\mathcal{G P}(\xi)}$, then the following statements are equivalent:

(1) $\mathcal{G P}(\xi)$-res.dim $M \leq m$.

(2) $\Omega^{n}(M) \in \mathcal{G P}(\xi)$ for all $n \geq m$.

(3) $\Omega_{\mathcal{G P}(\xi)}^{n}(M) \in \mathcal{G P}(\xi)$ for all $n \geq m$.

(4) $\xi x t_{\tilde{\xi}}^{n}(M, H)=0$ for all $n>m$ and all $P \in \mathcal{P}(\xi)$

(5) $\xi x t_{\xi}^{n}(M, L)=0$ for all $n>m$ and all $L \in \widehat{\mathcal{P}(\xi)}$.

(6) $\quad M$ admits a $\mathcal{G P}(\xi)$-approximation $\varphi: X \rightarrow M$, where $\varphi$ is a $\xi$-defaltion, such that there is an $\mathbb{E}$-triangle $K \longrightarrow X \stackrel{\varphi}{\longrightarrow} M->$ satisfying $\xi$-pd $K \leq m-1$.

(7) There are two $\mathbb{E}$-triangles

$$
W_{M} \rightarrow X_{M} \rightarrow M->
$$

and

$$
M \rightarrow W^{M} \longrightarrow X^{M}->
$$

in $\xi$ such that $X_{M}$ and $X^{M}$ are in $\mathfrak{X}, \xi$-pd $W^{M}=\mathcal{G P}(\xi)$-res.dim $W^{M} \leq m$ and $\xi$-pd $W_{M} \leq$ $m-1$.

Author Contributions: Writing—original draft preparation, L.T.; writing—review and editing, L.T. and L.L. All authors have read and agreed to the published version of the manuscript.

Funding: This research was funded by the project ZR2019QA015 supported by Shandong Provincial Natural Science Foundation.

Institutional Review Board Statement: Not applicable.

Informed Consent Statement: Not applicable.

Data Availability Statement: Not applicable.

Acknowledgments: The authors thank the referees for the helpful suggestions.

Conflicts of Interest: The authors declare no conflict of interest. 


\section{References}

1. Auslander, M.; Bridger, M. Stable Module Theory; American Mathematical Society: Providence, RI, USA, 1969.

2. Auslander, M.; Reiten, I. Applications of Contravariantly finite subcategories. Adv. Math. 1991, 86, 111-152. [CrossRef]

3. Auslander, M.; Buchweitz, R.O. The homological theory of maximal Cohen-Macaulay approximations. Mem. Soc. Math. France 1989, 38, 5-37. [CrossRef]

4. Zhu, X. Resolving resolution dimensions. Algebr. Represent. Theor. 2013, 16, 1165-1191. [CrossRef]

5. Huang, Z. Homological dimensions relative to preresolving subcategories. Kyoto J. Math. 2014, 54, 727-757. [CrossRef]

6. Ma, X.; Zhao, T. Resolving resolution dimensions in triangulated categories. Open Math. Available online: http://web.qfnu.edu. cn/tiweizhao/zh_CN/zdylm/29102/list/index.htm (accessed on 27 April 2021).

7. Ma, X.; Zhao, T.; Huang, Z. Resolving subcategories of triangulated categories and relative homological dimension. Acta Math. Sin. Engl. Ser. 2017, 33, 1513-1535. [CrossRef]

8. Hu, J.; Zhang, D.; Zhou, P. Gorenstein homological dimensions for extriangulated categories. Bull. Malays. Math. Sci. Soc. 2021, 1-18. [CrossRef]

9. Huang, Z. Proper resolutions and Gorenstein categories. J. Algebra 2013, 393, 142-169. [CrossRef]

10. Song, W.; Zhao, T.; Huang, Z. Homological dimensions relative to special subcategories. Algebra Colloq. 2021, $28,131-142$. [CrossRef]

11. Zhang, H.; Zhu, X. Resolving resolution dimension of recollements of abelian categories. J. Algebra Appl. 2020, 2150179. [CrossRef]

12. Nakaoka, H.; Palu, Y. Extriangulated categories, Hovey twin cotorsion pairs and model structures. Cah. Topol. Geom. Differ. Categ. 2019, LX-2, 117-193.

13. Hu, J.; Zhang, D.; Zhou, P. Proper classes and Gorensteinness in extriangulated categories. J. Algebra 2020, 551, 23-60. [CrossRef]

14. Hu, J.; Zhang, D.; Zhou, P. Proper resolutions and Gorensteinness in extriangulated categories. Front. Math. China 2021, 16, 95-117. [CrossRef]

15. Zhao, T.; Huang, Z. Phantom ideals and cotorsion pairs in extriangulated categories. Taiwanese J. Math. 2019, 23, 29-61. [CrossRef]

16. Zhao, T.; Tan, L.; Huang, Z. A bijection triangle in extriangulated categories. J. Algebra 2021, 574, 117-153. [CrossRef]

17. Available online: https:/ / arxiv.org/abs/1605.05607v4 (accessed on 27 April 2021).

18. Bühler, T. Exact categories. Expo. Math. 2010, 28, 1-69. [CrossRef]

19. Di, Z.; Wang, P. On finite resolutions in triangulated categories. Bull. Malays. Math. Sci. Soc. 2020, 43, 619-640. [CrossRef] 